\title{
CONVEXITY-PRESERVING FLUX IDENTIFICATION FOR SCALAR CONSERVATION LAWS MODELLING SEDIMENTATION
}

\author{
RAIMUND BÜRGER ${ }^{\mathrm{A}}$ AND STEFAN DIEHL ${ }^{\mathrm{B}, *}$
}

\begin{abstract}
The sedimentation of a suspension of small particles dispersed in a viscous fluid can be described by a scalar, nonlinear conservation law, whose flux function usually has one inflection point. The identification of the flux function is a problem of theoretical interest and practical importance for the implementation of plant-scale simulators of continuous sedimentation. For a real suspension, the Kynch test and the Diehl test, which are based on an initially homogenous suspension either filling the whole settling column or being initially located above clear liquid, respectively, provide data points that represent curved (convex or concave, respectively) suspension-supernate interfaces from which it is possible to reconstruct portions of the flux function to either side of the inflection point. Several functional forms can be employed to generate a provably convex or concave, twice differentiable accurate approximation of these data points via the solution of a constrained least-squares minimization problem. The resulting spline-like estimated trajectory can be converted into an explicit formula for the flux function. It is proved that the inverse problem of flux identification solved this way has a unique solution. The problem of gluing together the portions of the flux function from the Kynch and Diehl tests is addressed. Examples involving synthetic data are presented.
\end{abstract}

Mathematics subject classification: 35L65, 35R30, 65M32

\section{INTRODUCTION}

1.1. Scope. The sedimentation of small particles dispersed in a viscous fluid under the influence of a (mostly gravitational) body force is a process of theoretical and practical interest that appears as a controlled unit operation in mineral processing, wastewater treatment, the pulp-and-paper and chemical industry, medicine, volcanology, and other areas where a suspension must be separated into a clarified liquid and concentrated sediment. The particles are small compared with typical length scales (diameter and depth) of the settling vessel. Moreover, sedimentation models for these applications should be able to predict the behaviour of a given unit on relatively large temporal and spatial scales, and cover the full range of concentrations from the dilute limit to the packed sediment bed. These considerations

Date: September 2, 2012

Key words and phrases. inverse problem, nonlinear hyperbolic partial differential equation, explicit representation, batch sedimentation.

${ }^{*}$ Corresponding author.

${ }^{\mathrm{A}} \mathrm{CI}^{2} \mathrm{MA}$ and Departamento de Ingeniería Matemática, Facultad de Ciencias Físicas y Matemáticas, Universidad de Concepción, Casilla 160-C, Concepción, Chile. E-Mail: rburger@ing-mat.udec.cl.

${ }^{B}$ Centre for Mathematical Sciences, Lund University, P.O. Box 118, S-221 00 Lund, Sweden. E-Mail: diehl@maths.lth.se. 
justify representing the liquid and the solid particles as superimposed continuous phases, namely a liquid phase and one or several solid phases.

Most mathematical models for the simulation and control of sedimentation processes that incorporate these properties go back to the kinematic sedimentation model by Kynch [44], also known as the solids-flux theory, which is based on the following constitutive assumption. We consider batch sedimentation of an ideal suspension of monosized particles that show no compressive behaviour. The sedimentation takes place in a cylindrical vessel of height $H$. Let $\phi(x, t)$ be the local concentration (or volume fraction) of solids at height $x$, measured from the bottom of the vessel, and at time $t$. For ideal suspensions, the constitutive assumption by Kynch states that the settling velocity $v_{\mathrm{s}}$ of the particles depends on the local concentration $\phi$ only; $v_{\mathrm{s}}=v_{\mathrm{s}}(\phi) \geq 0$. The function $v_{\mathrm{s}}$ is assumed to satisfy $v_{\mathrm{s}} \in C^{2}$, $v_{\mathrm{s}}^{\prime}(\phi) \leq 0$ for $0 \leq \phi \leq \phi_{\max }$, and $v_{\mathrm{s}}\left(\phi_{\max }\right)=0$, where $\phi_{\max }$ is the maximum packing concentration. Thus, the evolution of $\phi$ as a function of $x$ and $t$ is governed by the scalar conservation law with nonlinear flux

$$
\frac{\partial \phi}{\partial t}-\frac{\partial f_{\mathrm{b}}(\phi)}{\partial x}=0, \quad f_{\mathrm{b}}(\phi):=\phi v_{\mathrm{s}}(\phi),
$$

supplied with suitable initial and boundary conditions. (The unconventional negative sign for the flux term originates from our upwards pointing $x$-axis, which is opposite to the flux direction.)

The so-called batch-settling flux $f_{\mathrm{b}}$, or equivalently, the function $v_{\mathrm{s}}$, reflects specific material properties of the material under study. In most circumstances, $f_{\mathrm{b}}$ is convex-concave with one inflection point $\phi_{\text {infl. }}$. The same function $f_{\mathrm{b}}$ also appears in extensions of (1) to models of continuous sedimentation. The reliable identification of the function $f_{\mathrm{b}}$ from experimental data is therefore of considerable practical and theoretical interest. Entropy solutions of (1) for a constant or piecewise constant initial concentration can be constructed explicitly. Specific curved discontinuities (shocks) of such solutions, corresponding to curves in the standard height versus time settling plot, arise from an explicit transformation of a concave or convex portion of $f_{\mathrm{b}}$. Physically, these trajectories correspond to the temporal evolution of the suspension-supernate interface. This interface is fairly easy to observe, and its measurement has been documented in numerous papers in literature; e.g. [16, $18,29,38,42,47,48,51,56,59]$.

The purpose of this paper is to introduce a technique that permits to reconstruct the aforementioned convex or concave portions of $f_{\mathrm{b}}$ (located to the right and left of $\phi_{\text {inf }}$, respectively) from measurements of curved suspension-supernate interfaces and to obtain explicit formulas for the estimation of these portions of $f_{\mathrm{b}}$. One curved interface separates the suspension from the supernatant liquid in a standard batch-settling test of an initially homogeneous suspension (the Kynch test). Another curved interface separates the fan of characteristics forming a transsonic rarefaction wave emerging from the interface between a column of a highly concentrated suspension that is initially located above, and initially separated by a membrane from, a column of clear liquid (the Diehl test). The main novelty lies in the technique of approximating the height versus time data points by representing the interface (but affected by errors in measurement, etc.) by a smooth function that is defined piecewise by either quadratic polynomials, cubic polynomials, or rational functional forms. The salient feature of the present approach is the particular approximation of the data points by the solution of a constrained optimization 
problem, which ensures that the identified curved interface is $C^{2}$ and either convex or concave, and that the corresponding portion of $f_{\mathrm{b}}$ has the same property. The Kynch and Diehl tests can be conducted independently for the same material, and the problem of properly "gluing" together the separate portions of $f_{\mathrm{b}}$ identified by either test is made so that a final explicit formula for the estimation of $f_{\mathrm{b}}$ is obtained.

1.2. Related works. Inverse problems for scalar conservation laws of type (1), where (a portion of) the flux function $f_{\mathrm{b}}$ (or $-f_{\mathrm{b}}$ ) is identified without any assumed parametric form, can be found in [30, 40, 41, 53]. James and Sepúlveda [40] analyze the minimization of a nonstandard cost function which contains the difference between observed data and the solution of (1) at a fixed time point. This is done via an adjoint equation obtained from a formal computation of the gradient of the cost function, in spite of the fact that the latter may not be differentiable. The nonuniqueness of identifying $f_{\mathrm{b}}$ when the direct problem contains discontinuities still remains, and in a particular example the authors resolve this by assuming a physically motivated parametric function for $f_{\mathrm{b}}$. Kang and Tanuma [41] deal with the case when $-f_{\mathrm{b}}^{\prime \prime}(\phi)>C>0$ for some constant $C$ and the identification of $f_{\mathrm{b}}$ is made from observations of the shock formed for large time for initial data with compact support. Fernández-Berdaguer and Savioli [30, 53] consider the displacement of oil by water in petroleum reservoirs where the oil fractional flux function is to be identified. This is done with a relatively advanced method including a least-squares technique in functional spaces. Their method is successful in their presented examples; however, they need to start with a continuous solution of the direct problem (1) for small times and hence need to assume that the initial datum is smooth. This is not satisfied in our sedimentation problems.

To put the paper into the proper perspective with respect to sedimentation, we mention that formulas equivalent to $v_{\mathrm{s}}=v_{\mathrm{s}}(\phi)$ were derived in the dilute limit $\phi / \phi_{\max } \ll 1$ more than a century ago by A. Einstein [27], and in the 1940s for both dilute and concentrated suspensions (see, e.g., [37, 54, 55, 58]). It was Kynch's specific contribution [44] that he explicitly solved the governing equation (1) for initially constant concentrations. His arguments concerning characteristics, discontinuities, and their possible intersections led to solutions that coincide with those singled out by the current entropy solution theory for scalar hyperbolic conservation laws. Historical accounts and overviews related to sedimentation models based on (1) are provided in $[10,24,25]$.

Of particular importance is the extension of (1) to models of continuous sedimentation in secondary settling tanks (SSTs) or clarifier-thickener units handling suspensions forming compressible sediments. For that application the governing equation (1) is replaced by a degenerate parabolic equation with discontinuous coefficients, which are however still based on knowledge of $f_{\mathrm{b}}$. We refer to $[5,6,7,8,19,20]$ for the mathematical and numerical analysis of these models (this list is incomplete). During normal operation of SSTs, it is well known that solutions of the model PDE contain concentrations in the convex part of the flux function $f_{\mathrm{b}}$. During transient solutions with a highly loaded SST at the limit to overloaded situations, the concave part of $f_{\mathrm{b}}$ influences the solution; see [22]. An accurate determination of the concave part of $f_{\mathrm{b}}$ is therefore crucial for the saturating bounds of the regulator for SSTs presented in [23]. 
The problem of determining the flux $f_{\mathrm{b}}$ for a given material has been solved in several ways by using measurements from the standard Kynch test; see the references in [21]. Roughly speaking, one approach consists in choosing a global parametric form for $f_{\mathrm{b}}$ with a vector of a small number of parameters. One then compares an observed temporal or spatial solution profile with a numerically simulated one, and seeks to minimize the distance between both by successively varying the parameter vector. One can employ a variety of descent methods to determine the direction of the parameter correction (which should be aligned with the gradient of the cost functional) [3, 4]; another technique is based on solving an adjoint problem [14]. These techniques are most common when there is an additional degenerate diffusive term accounting for sediment compressibility, and exact closed-form solutions of the direct problem are not at hand.

Alternatively, one may identify certain observed trajectories with portions of an exact solution, and determine the flux parameters by a constrained least-squares technique. This procedure does not require the numerical solution of PDEs. Neither does the graphical estimation method presented by Kynch [44]. For the Kynch test available treatments pursuing such approaches include $[2,26,31,32,33,34,35,36$, $45,60,61]$.

The graphical method by Kynch utilizes the PDE theory connection between the flux function and the curved discontinuity of the Kynch test. An explicit formula for the estimated flux function in terms of measurable variables was presented by Lester et al. [45]. However, their formula contains an integral over the measured settling velocities. Diehl [21] presented a simpler formula, which has been used by Grassia et al. [35] and is utilized herein together with a constrained least-squares method to obtain closed-form estimations for $f_{\mathrm{b}}$. In Section 7 some of the previous treatments will be compared with ours in a more detailed way.

With the aim of a new batch-settling experiment, called the Diehl test, a portion of the concave part of the flux function can also be estimated with explicit formulas (Diehl [21]). The experiment requires a special arrangement, since a highly concentrated suspension should initially be applied on top of clear liquid. On the other hand, the solution of the PDE is more involved with a richer behaviour than the Kynch test. This initial datum has also been used for simulation and parameter identification elsewhere; see e.g. [3, 4].

1.3. Outline of the paper. The remainder of this paper is organized as follows. In Section 2 we first describe the direct problem, namely the initial-boundary value problem (IBVP) for (1) that models batch sedimentation (Section 2.1). We then recall in Section 2.2 the construction of the unique solution of the IBVP for a constant initial datum $\phi_{0}$, and specify the range of initial concentrations $\phi_{0}$ for which the solution exhibits a lower rarefaction fan (needed for flux identification). The present flux identification method is based on a pair of representation formulas due to Diehl [21], which expresses the pairs of values $\left(\phi, f_{\mathrm{b}}(\phi)\right)$ assumed within the rarefaction fan (in a sense that will be made precise) as an explicit function of pairs $(t, h(t))$, where $h(t)$ is the position of the curved interface at time $t$. This pair of representation formulas is introduced in Section 2.3, along with an alternative pair due to Kunik [43] that expresses $(t, h(t))$ as an explicit function of $\left(\phi, f_{\mathrm{b}}(\phi)\right)$. In that section it is also shown that if Diehl's formulas are applied to an estimated portion of the curved interface $\breve{h}(t)$ with $\breve{h} \in C^{2}$ and if $\breve{h}$ is strictly convex, then the resulting estimated portion of $f_{\mathrm{b}}$, denoted by $\breve{f}_{\mathrm{b}}$, is also $C^{2}$ and strictly convex. 
In Section 3 we describe how the "raw" data typically available from measurements of a Kynch test, namely data points $\left(t_{j}, x_{j}\right)$ that represent the curved discontinuity (Section 3.1), give rise to a once or twice continuously differentiable and convex curve $\breve{h}(t)$ that estimates the real curved interface. This is done by representing $\breve{h}(t)$ by piecewise quadratic, piecewise cubic, or particular piecewise rational functional forms per segment, which give rise to the "quadratic-fit", "spline-fit" and "special-fit" methods, respectively. Convexity conditions for each of these methods are identified along with certain auxiliary functions appearing in the Diehl formula. While Section 3 is focused on the functional forms, Section 4 deals with the actual solution of the inverse problem, namely of the quadratic programming problem of computing the coefficients appearing in $\breve{h}(t)$ such that the resulting function is globally convex. An optimization problem is formulated for all three methods (Section 4.1), and it is proved that the problem has a unique solution (Section 4.2). In Section 4.4, we present the application of the flux identification techniques to synthetically produced Kynch-test data.

In Section 5, we summarize the steps and modifications necessary for the analogous treatment of the curved supernate-suspension interface arising in the Diehl test. This interface is concave, and its reconstruction provides an estimate of the concave portion of $f_{\mathrm{b}}$. This is illustrated with synthetic data in Section 5.1. Combining the treatments of both the Kynch and Diehl tests for one and the same material, one is able to reconstruct the portions of $f_{\mathrm{b}}$ to either side of $\phi_{\text {infl }}$. In Section 6 , we describe how to complement the two estimated portions of $f_{\mathrm{b}}$ to obtain an entire flux function. Conclusions are summarized in Section 7.

\section{The KynCh MOdel of BAtCh SEDimentation: The DiRECT PROBlEM}

2.1. The initial-boundary-value problem. We use the convention that the settling velocity $v_{\mathrm{s}}$ and the flux $f_{\mathrm{b}}$ are positive in the downward direction, which is opposite to the direction of the $x$-axis. The batch-settling flux $f_{\mathrm{b}}$ has generally inflection points. For simplicity, we consider here only the case of one inflection point $\phi_{\text {infl }}$, which necessarily satisfies $f_{\mathrm{b}}^{\prime}\left(\phi_{\mathrm{infl}}\right)<0$; see Figure 1 (left). Our analysis will use results by Diehl [21], where the case when $f_{\mathrm{b}}$ has two inflection points is also covered. The identification method presented here can in principle be applied to a flux function with several inflection points. The $\phi$-intervals of estimation will then be a subset of the union of the convex (concave) parts $f_{\mathrm{b}}$ (cf. the "convex hull" of $f_{\mathrm{b}}$ ), and special care has to be taken for the corresponding convex (concave) continuous discontinuity where its slope make jumps. We leave the details.

The conservation of mass implies an initial-value zero-boundary-flux problem. For a piecewise smooth initial-value function $\phi_{\text {init }}(x)$ that satisfies $\phi_{\text {init }}\left(H^{-}\right)<$ $\phi_{\max }$ and $\phi_{\text {init }}\left(0^{+}\right)>0$ (one-sided limits), an entropy condition at the boundaries (Diehl $[19$, Section 5]) yields the initial-boundary-value problem

$$
\begin{array}{ll}
\frac{\partial \phi}{\partial t}-\frac{\partial f_{\mathrm{b}}(\phi)}{\partial x}=0, & 0<x<H, t>0, \\
\phi(x, 0)=\phi_{\text {init }}(x), & 0<x<H, \\
\phi\left(0^{+}, t\right)=\phi_{\max }, & t>0, \\
\phi\left(H^{-}, t\right)=0, & t>0 .
\end{array}
$$

In a standard batch-settling experiment, here called the Kynch test, it is assumed that the suspension is initially homogeneous, i.e. $\phi_{\text {init }}(x) \equiv \phi_{0}$, and in an alternative 

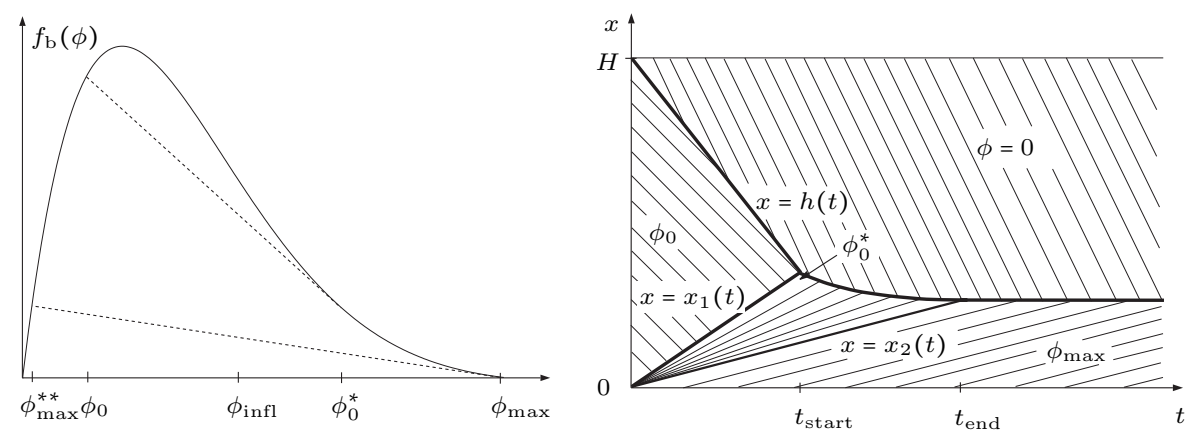

Figure 1. Left: The batch-settling flux function. The dotted line through the point $\left(\phi_{\max }, 0\right)$ has the slope $f_{\mathrm{b}}^{\prime}\left(\phi_{\max }^{* *}\right)$. Right: A schematic solution of a Kynch test with $\phi_{0} \in\left(\phi_{\max }^{* *}, \phi_{\max }\right]$. Thin lines are characteristics and thick lines discontinuities, except for $x_{2}$, which is a line of continuity. The solution assumes values in $\left\{\phi_{0}\right\} \cup\left[\phi_{0}^{*}, \phi_{\max }\right]$.

batch-settling test, called the Diehl test [21], there is initially a high concentration $\phi_{0}$ in a top layer, below which there is liquid. A solution of (2) is then piecewise smooth. For a function $f_{\mathrm{b}}$ with exactly one inflection point there are three qualitatively different solutions of the Kynch test, called modes of sedimentation, depending on the value of $\phi_{0}$; for exactly two inflection points, there are in total seven modes (under the assumption that the settling velocity $v_{\mathrm{s}}$ is a decreasing function; see [21]). These modes of sedimentation are derived in detail in [11] and are summarized in [9]. (In the terminology of [9, 11], the choice of $\phi_{0}$, considered herein for the Kynch test, corresponds to modes of sedimentation MS-2 and MS-3.)

Across each smooth curve of discontinuity $x=x(t)$, between the values $\phi_{ \pm}:=$ $\phi(x(t) \pm 0, t)$, the jump condition (also known as Rankine-Hugoniot condition; and which is a mere consequence of the conservation of mass)

$$
-x^{\prime}(t)=\frac{f_{\mathrm{b}}\left(\phi_{+}\right)-f_{\mathrm{b}}\left(\phi_{-}\right)}{\phi_{+}-\phi_{-}}=: S\left(\phi_{+}, \phi_{-}\right)
$$

holds. The solution is unique if every discontinuity satisfies the following entropy condition by Oleinik [50]:

$$
S\left(\phi_{+}, u\right) \geq S\left(\phi_{+}, \phi_{-}\right) \quad \text { for all } u \text { between } \phi_{+} \text {and } \phi_{-},
$$

where (for convenience) we define $S(\phi, \phi):=f_{\mathrm{b}}^{\prime}(\phi)$.

2.2. Construction of the solution. We need the following operations for the construction of the solution of (2) (cf. Ballou [1]). Given $\phi \in\left[0, \phi_{\max }\right]$, we define

$$
\begin{aligned}
\phi^{*} & :=\max \left\{u \in\left[\phi, \phi_{\max }\right]: f_{\mathrm{b}}^{\prime}(u)=S(\phi, u) \leq S(\phi, v) \forall v \in[\phi, u]\right\}, \\
\phi_{*} & :=\min \left\{u \in[0, \phi]: f_{\mathrm{b}}^{\prime}(u)=S(\phi, u) \leq S(\phi, v) \forall v \in[u, \phi]\right\}, \\
\phi^{* *} & :=\min \left\{u \in[0, \phi]: u^{*}=\phi\right\} .
\end{aligned}
$$

The solution of $(2)$ for $\phi_{0} \in\left(\phi_{\max }^{* *}, \phi_{\max }\right]$ is shown in Figure 1 (right). The line $x=x_{1}(t)$ is a discontinuity if $\phi_{0} \in\left(\phi_{\max }^{* *}, \phi_{\mathrm{inf}}\right)$, otherwise it is a line of continuity. 
If $0<\phi_{0} \leq \phi_{\max }^{* *}$, then the lines $x=x_{1}(t)$ and $x=x_{2}(t)$ in Figure 1 (right) coincide, $t_{\text {start }}=t_{\text {end }}$ and there is no expansion wave. In the present paper we consider only the case $\phi_{0} \in\left(\phi_{\max }^{* *}, \phi_{\max }\right)$, i.e. when there is a non-trivial expansion wave.

We denote the height of the uppermost discontinuity between zero and nonzero concentrations by $h(t)$. According to (3), the function $h(t)$ satisfies

$$
-h^{\prime}(t)=\frac{f_{\mathrm{b}}\left(\phi_{0}\right)}{\phi_{0}}=v_{\mathrm{s}}\left(\phi_{0}\right), \quad 0<t<t_{\text {start }},
$$

where $t_{\text {start }}$ is the time of intersection with another discontinuity, namely $x=x_{1}(t)=$ $-f_{\mathrm{b}}^{\prime}\left(\phi_{0}^{*}\right) t$, originating from the bottom. Between $x_{1}(t)$ and down to the line of continuity $x=x_{2}(t)=-f_{\mathrm{b}}^{\prime}\left(\phi_{\max }\right) t$ there is an expansion wave of continuously increasing concentrations from $\phi_{0}^{*}$ to $\phi_{\max }$. The solution $\phi$ in the expansion wave satisfies

$$
\frac{x}{t}=-f_{\mathrm{b}}^{\prime}(\phi(x, t)) \quad(=\text { the slopes of the characteristics }) .
$$

If $f_{\mathrm{b}}^{\prime}\left(\phi_{\max }\right)<0$, then the line $x_{2}(t)$ intersects $x=h(t)$ at the time $t_{\mathrm{end}}$, after which the solution is stationary. If $f_{\mathrm{b}}^{\prime}\left(\phi_{\max }\right)=0$, then $\phi_{\max }^{* *}=0$ and $x_{2}(t) \equiv 0$, i.e. the expansion wave fills out the region down to the $t$-axis $\left(t_{\mathrm{end}}=\infty\right)$. Since the derivative $h^{\prime}$ may be discontinuous at $t_{\text {start }}$, we use the convention $h^{\prime}\left(t_{\text {start }}\right):=h^{\prime}\left(t_{\text {start }}^{+}\right)$.

2.3. Diehl's and Kunik's representation formulas. Given the upper curved discontinuity $h(t), t \in\left[t_{\text {start }}, t_{\text {end }}\right]$, of the solution of (2), Kynch [44] presented a graphical procedure for obtaining the flux function $f_{\mathrm{b}}$ in the interval $\left[\phi_{0}^{*}, \phi_{\max }\right]$ (the "tail"). Diehl [21] showed that Kynch's graphical procedure can be written by representation formulas; namely the tail of the flux function can be expressed as a function of the curved discontinuity $h$ and its derivative $h^{\prime}$. This is a solution of the inverse problem of obtaining (the tail of) the flux function $f_{\mathrm{b}}$ given the solution of (2). Kunik [43] presented representation formulas for the global solution of the forward problem (2) for a monotone initial-value function $\phi_{\text {init }}$. In the special batch-sedimentation case where $\phi_{\text {init }} \equiv \phi_{0}$, Kunik's formulas relate the curved discontinuity $h$ as a function of the flux function $f_{\mathrm{b}}$ in precisely the same way as Diehl's formulas relate $f_{\mathrm{b}}$ as a function of $h$. We will here explore this symmetry.

We denote the concentration just below the curved discontinuity by

$$
\phi_{h}(t):=\phi\left(h(t)^{-}, t\right) \quad \text { for } t_{\text {start }} \leq t \leq t_{\mathrm{end}},
$$

where $\phi_{h}$ is an increasing $C^{1}$ function that maps the interval $\left[t_{\text {start }}, t_{\text {end }}\right]$ to $\left[\phi_{0}^{*}, \phi_{\max }\right]$. In the rest of this section we restrict the two functions $h$ and $f_{\mathrm{b}}$ to these two intervals. Evaluating (6) at the discontinuity $x=h(t)$ and inserting (7) we get

$$
\frac{h(t)}{t}=-f_{\mathrm{b}}^{\prime}\left(\phi_{h}(t)\right), \quad \text { for } t_{\mathrm{start}} \leq t \leq t_{\mathrm{end}} .
$$

The jump condition (3) for $x=h(t), t_{\text {start }} \leq t \leq t_{\text {end }}$, implies that

$$
-h^{\prime}(t)=\frac{f_{\mathrm{b}}\left(\phi_{h}(t)\right)}{\phi_{h}(t)} \text { for } t_{\mathrm{start}} \leq t \leq t_{\mathrm{end}}
$$

Since $\phi \in C^{1}$ and $f_{\mathrm{b}} \in C^{2},(9)$ implies that $h \in C^{2}$ and $h^{\prime}<0$. Differentiating (8), we get

$$
\phi_{h}^{\prime}(t)=-\frac{h^{\prime}(t)+f_{\mathrm{b}}^{\prime}\left(\phi_{h}(t)\right)}{t f_{\mathrm{b}}^{\prime \prime}\left(\phi_{h}(t)\right)}>0 .
$$


Furthermore, differentiating (9), we get (for $t_{\text {start }} \leq t \leq t_{\text {end }}$ )

$h^{\prime \prime}(t)=-\frac{f_{\mathrm{b}}^{\prime}\left(\phi_{h}(t)\right) \phi_{h}^{\prime}(t) \phi_{h}(t)-f_{\mathrm{b}}\left(\phi_{h}(t)\right) \phi_{h}^{\prime}(t)}{\phi_{h}(t)^{2}}=-\frac{\phi_{h}^{\prime}(t)}{\phi_{h}(t)}\left(f_{\mathrm{b}}^{\prime}\left(\phi_{h}(t)\right)-v_{\mathrm{s}}\left(\phi_{h}(t)\right)\right)$.

Since $f_{\mathrm{b}}^{\prime}\left(\phi_{h}(t)\right)<0$ and the other functions are positive, there holds $h^{\prime \prime}(t)>0$. Thus, $f_{\mathrm{b}}$ and $h$ are both decreasing, strictly convex and $C^{2}$ functions (as restrictions to the intervals of interest).

Let us now define the following auxiliary functions:

$$
\begin{aligned}
\eta(t) & :=h(t)-t h^{\prime}(t), \\
\Phi(\phi) & :=f_{\mathrm{b}}(\phi)-\phi f_{\mathrm{b}}^{\prime}(\phi) .
\end{aligned}
$$

Since $\eta>0, \Phi>0, \eta^{\prime}(t)=-t h^{\prime \prime}(t)<0$ and $\Phi^{\prime}(\phi)=-\phi f_{\mathrm{b}}^{\prime \prime}(\phi)<0$ on the intervals of interest, both $\eta$ and $\Phi$ are invertible. Adding equations (8) and (9), we get

$$
\frac{h(t)}{t}-h^{\prime}(t)=-f_{\mathrm{b}}^{\prime}\left(\phi_{h}(t)\right)+\frac{f_{\mathrm{b}}\left(\phi_{h}(t)\right)}{\phi_{h}(t)},
$$

which (by (10) and (11)) is equivalent to $\phi_{h}(t) \eta(t)=t \Phi\left(\phi_{h}(t)\right)$. The expressions on both sides are actually constant and equal to the total mass per unit area, i.e.,

$$
\phi_{h}(t) \eta(t)=H \phi_{0}=t \Phi\left(\phi_{h}(t)\right) \text { for } t_{\text {start }} \leq t \leq t_{\text {end }} .
$$

This was proved by Diehl [21], utilizing (4), (8) and (9).

Remark 2.1. The left equality of (12) was in fact derived already by Kynch [44], who used the following physical argumentation together with (8) and (9). Consider a fixed time point $t \in\left(t_{\mathrm{start}}, t_{\mathrm{end}}\right)$ and the characteristic in the expansion wave between the origin and the point $(t, h(t))$. Along this characteristic, the concentration has the constant value $\phi_{h}(t)$ and the slope $-f_{\mathrm{b}}^{\prime}\left(\phi_{h}(t)\right)>0$, which is equal to the speed of the characteristic upwards. All particles pass this characteristic with the constant relative speed $v_{\mathrm{s}}\left(\phi_{h}(t)\right)-f_{\mathrm{b}}^{\prime}\left(\phi_{h}(t)\right)$ and, hence, constant flux (mass per unit area and unit time) given by

$$
\phi_{h}(t)\left[v_{\mathrm{s}}\left(\phi_{h}(t)\right)-f_{\mathrm{b}}^{\prime}\left(\phi_{h}(t)\right)\right]=\phi_{h}(t)\left(\frac{f_{\mathrm{b}}\left(\phi_{h}(t)\right)}{\phi_{h}(t)}-f_{\mathrm{b}}^{\prime}\left(\phi_{h}(t)\right)\right) .
$$

The total mass of the particles per unit area is $H \phi_{0}$. During the time period $[0, t]$ all particles have passed, which together with (8) and (9) yields

$$
H \phi_{0}=\phi_{h}(t)\left(\frac{f_{\mathrm{b}}\left(\phi_{h}(t)\right)}{\phi_{h}(t)}-f_{\mathrm{b}}^{\prime}\left(\phi_{h}(t)\right)\right) t=\phi_{h}(t)\left(-h^{\prime}(t)+\frac{h(t)}{t}\right) t,
$$

which in view of (10) is equivalent to $H \phi_{0}=\Phi\left(\phi_{h}(t)\right) t=\phi_{h}(t) \eta(t)$.

The following representation formulas by Diehl [21] are obtained by (12) and(9). The parametrization of the tail of the flux function is

$$
\left\{\begin{aligned}
\phi & =\frac{H \phi_{0}}{\eta(t)} \\
f_{\mathrm{b}}(\phi) & =-\frac{H \phi_{0}}{\eta(t)} h^{\prime}(t)
\end{aligned} \quad \text { for } t_{\text {start }} \leq t \leq t_{\mathrm{end}} .\right.
$$

Since $\eta$ is invertible, we may solve the first equation in (13) for $t$ and insert the result into the second to obtain the explicit formula

$$
f_{\mathrm{b}}(\phi)=-\phi h^{\prime}\left(\eta^{-1}\left(\frac{H \phi_{0}}{\phi}\right)\right) \quad \text { for } \phi_{0}^{*} \leq \phi \leq \phi_{\max }, \text { where } \phi_{0}^{*}=\frac{H \phi_{0}}{\eta\left(t_{\text {start }}\right)} .
$$


The representation formulas by Kunik [43] are obtained by (12) and (8):

$$
\left\{\begin{aligned}
t & =\frac{H \phi_{0}}{\Phi(\phi)}, \\
h(t) & =-\frac{H \phi_{0}}{\Phi(\phi)} f_{\mathrm{b}}^{\prime}(\phi)
\end{aligned} \quad \text { for } \phi_{0}^{*} \leq \phi \leq \phi_{\max } .\right.
$$

Since $f_{\mathrm{b}}$ is invertible, we may solve the first equation in (15) for $\phi$ to obtain the explicit formula

$$
h(t)=-t f_{\mathrm{b}}^{\prime}\left(\Phi^{-1}\left(\frac{H \phi_{0}}{t}\right)\right), \quad \text { for } t_{\text {start }} \leq t \leq t_{\text {end }} .
$$

Given an estimated curved discontinuity $\breve{h}$, we may define an estimated flux function $\breve{f}_{\mathrm{b}}$ via (13). Then these functions satisfy (9), but it is not obvious that they satisfy (8). Hence, there is no direct information on $\breve{f}_{\mathrm{b}}^{\prime}$ and whether the regularity of $\breve{f}_{\mathrm{b}}$ is equal to that of $\breve{h}$. The following lemma and theorem resolve this issue.

Lemma 2.1. Assume that $h, f_{\mathrm{b}} \in C^{2}$ are decreasing and strictly convex functions. Then Equations (13) and (15) are equivalent.

Proof. We have $h \in C^{2}$ and hence $\eta, \eta^{-1} \in C^{1}$. The first equality of (13) is equivalent to

$$
t=\eta^{-1}\left(\frac{H \phi_{0}}{\phi}\right)=: t(\phi), \quad t_{\text {start }} \leq t \leq t_{\mathrm{end}}, \quad \phi_{0}^{*} \leq \phi \leq \phi_{\max },
$$

where $t(\cdot) \in C^{1}$. Differentiating $\phi \eta(t(\phi))=H \phi_{0}$, see (12), with respect to $\phi$, we get

$$
\left.\eta(t(\phi))+\phi\left(-t h^{\prime \prime}(t(\phi))\right) t^{\prime}(\phi)=0 \quad \Longleftrightarrow \quad-\phi h^{\prime \prime}(t(\phi))\right) t^{\prime}(\phi)=-\frac{\eta(t(\phi))}{t(\phi)}
$$

Writing the second equality of (13) as $f_{\mathrm{b}}(\phi)=-\phi h^{\prime}(t(\phi))$, differentiating it and using (17), we obtain

$$
f_{\mathrm{b}}^{\prime}(\phi)=-h^{\prime}(t(\phi))-\phi h^{\prime \prime}(t(\phi)) t^{\prime}(\phi)=-h^{\prime}(t(\phi))-\frac{\eta(t(\phi))}{t(\phi)}=-\frac{h(t(\phi))}{t(\phi)} .
$$

Now we get the first equation of (15) from

$$
t \Phi(\phi)=t\left(f_{\mathrm{b}}(\phi)-\phi f_{\mathrm{b}}^{\prime}(\phi)\right)=t\left(-\phi h^{\prime}(t)+\phi \frac{h(t)}{t}\right)=\phi \eta(t)=H \phi_{0}
$$

and then the second from (18). By the symmetry between (13) and (15), one could start with the latter equations and derive the former in the same way.

In Section 3, we will use functions $\breve{h}$ consisting of piecewise highly regular functions with the regularity $C^{1}$ or $C^{2}$ at the fitting points.

Theorem 2.1. Assume that the function $\breve{h} \in C^{k}$, for some $k \geq 1$, satisfies $\breve{h}>0$ and $\breve{h}^{\prime}<0$, such that $\breve{\eta}(t):=\breve{h}(t)-t \breve{h}^{\prime}(t)$ is decreasing. If $k=1$, we assume that $\breve{h} \in C^{2}$ except at a finite number of points. Then the estimated flux function defined by (13) satisfies $\breve{f}_{\mathrm{b}} \in C^{k}$. Conversely, given $\breve{f}_{\mathrm{b}} \in C^{k}$, then $\breve{h} \in C^{k}$ holds.

Proof. For $k \geq 2$, the proof of Lemma 2.1 can be repeated with $C^{2}$ replaced by $C^{k}$ and $C^{1}$ replaced by $C^{k-1}$. Then (18) gives that $\breve{f}_{\mathrm{b}}^{\prime} \in C^{k-1}$, hence $\breve{f}_{\mathrm{b}} \in C^{k}$. For $k=1, \breve{h}^{\prime \prime}$ and $\breve{t}^{\prime}(\cdot)$ are not defined at a finite number of points. However, outside these finite number of points, (17) holds and states that the product $\breve{h}^{\prime \prime}(\breve{t}(\cdot)) \breve{t}^{\prime}(\cdot)$ is 
equal to a function, which is defined and continuous for all $\phi \in\left[\phi_{0}^{*}, \phi_{\max }\right]$. Hence, (18) also holds, so that $\breve{f}_{\mathrm{b}}^{\prime} \in C$, hence $\breve{f}_{\mathrm{b}} \in C^{1}$.

\section{Piecewise fitting of the Convex discontinuity of A Kynch test}

3.1. Preliminaries. Assume that we are given $N$ pairs of data points that represent the curved discontinuity in the interval $\left[t_{\text {start }}, t_{\text {end }}\right]$; see Figure 1 (right):

$$
\left(t_{j}, x_{j}\right), \quad j=1, \ldots, j_{2}, \ldots, j_{3}, \ldots, j_{n}, \ldots, N .
$$

Let $j_{1}:=1$ and $j_{n+1}:=N$. The number of data points belonging to each interval $\left(t_{j_{i}}, t_{j_{i+1}}\right]$ is $N_{i}:=j_{i+1}-j_{i}$, so that $N=1+\sum_{i=1}^{n} N_{i}$. We would like to fit smooth functions $\breve{h}_{i}, i=1, \ldots, n$ close to these data such that the graph of

$$
\breve{h}(t):=\sum_{i=1}^{n} \breve{h}_{i}(t) \chi_{i}(t) \quad \text { for } t_{1}<t \leq t_{N}
$$

where

$$
\chi_{i}(t)= \begin{cases}1 & \text { if } t_{j_{i}}<t \leq t_{j_{i+1}} \\ 0 & \text { otherwise, }\end{cases}
$$

is an estimation of the curved discontinuity of the function $h$ of the solution of the PDE. The goal is to substitute (20) into (14) to obtain an explicit formula for a portion of the batch settling flux function $\breve{f}_{\mathrm{b}}$.

The functions $\breve{h}_{i}$ will be chosen such that they are sufficiently smooth in a neighbourhood of the interval $\left(t_{j_{i}}, t_{j_{i+1}}\right)$. We present below three functional forms for $\breve{h}_{i}$, all with a linear dependence in the parameters, the number of which are either 3 or4. At the end points of the $n$ intervals, we require continuity in the function value and derivatives up to the number of parameters minus 2 . For $\breve{h} \in C^{2}$, each $\breve{h}_{i}$ contains four parameters, $a_{i}, b_{i}, c_{i}$ and $d_{i}$, and we require $\breve{h}$ to be twice continuously differentiable, which leads to the continuity constraints

$$
\begin{array}{ll}
\breve{h}_{i-1}\left(t_{j_{i}}\right)=\breve{h}_{i}\left(t_{j_{i}}\right), & i=2, \ldots, n, \\
\breve{h}_{i-1}^{\prime}\left(t_{j_{i}}\right)=\breve{h}_{i}^{\prime}\left(t_{j_{i}}\right), & i=2, \ldots, n, \\
\breve{h}_{i-1}^{\prime \prime}\left(t_{j_{i}}\right)=\breve{h}_{i}^{\prime \prime}\left(t_{j_{i}}\right), & i=2, \ldots, n .
\end{array}
$$

These constitute $3(n-1)$ equations of the $4 n$ parameters (in the 4 -parameter case). To obtain a unique solution of a least-squares minimization problem (see Theorem 4.1 in Section 4.2), it turns out that in each interval $\left(t_{j_{i}}, t_{j_{i+1}}\right]$, there should be at least as many data points as parameters, i.e., $j_{i+1}-j_{i} \geq 4$, which implies that the total number $N$ of data points should satisfy

$$
N=j_{n+1}=1+\sum_{i=1}^{n}\left(j_{i+1}-j_{i}\right) \geq 1+4 n .
$$

In accordance with the properties of the solution of (2), we require

$$
\begin{array}{ll}
\breve{h}_{i}^{\prime}(t)<0, \quad t_{j_{i}} \leq t<t_{j_{i+1}}, \quad i=1, \ldots, n, & \text { and } \quad \breve{h}_{n}^{\prime}\left(t_{N}\right) \leq 0, \\
\breve{h}_{i}^{\prime \prime}(t)>0, \quad t_{j_{i}} \leq t<t_{j_{i+1}}, \quad i=1, \ldots, n, & \text { and } \quad \breve{h}_{n}^{\prime \prime}\left(t_{N}\right) \geq 0,
\end{array}
$$

Because of the continuity constraint (22), we can conclude that $\breve{h}^{\prime}$ is increasing (in both 3- and 4-parameter cases). Hence, (24) can be replaced by the single constraint

$$
\breve{h}_{n}^{\prime}\left(t_{N}\right) \leq 0 \text {. }
$$


The parameters are determined as the solution of a least-squares optimization problem in Section 4, where the constraints are such that they imply (21)-(23) and (25)-(26). Once the optimization problem is solved, we may continue by defining $\breve{\eta}_{i}(t):=\breve{h}_{i}(t)-t \breve{h}_{i}^{\prime}(t), i=1, \ldots, n$. The continuity constraints $(21)-(22)$ imply that

$$
\breve{\eta}(t)=\breve{h}(t)-t \breve{h}^{\prime}(t)=\sum_{i=1}^{n} \breve{\eta}_{i}(t) \chi_{i}(t) \quad \text { for } t_{1}<t \leq t_{N} .
$$

The inverse of $\breve{\eta}$ can be written by means of a sum of the inverses of all $\breve{\eta}_{i}$. We have to transform the characteristic functions $\chi_{i}$. Since $\breve{\eta}$ is decreasing, it maps the interval $\left(t_{j_{i}}, t_{j_{i+1}}\right]$ to the interval $\left[\breve{\eta}\left(t_{j_{i+1}}\right), \breve{\eta}\left(t_{j_{i}}\right)\right)$. Because of the argument of $\eta^{-1}$ in (14), the corresponding interval for $\phi$ is

$$
\breve{\eta}\left(t_{j_{i+1}}\right) \leq \frac{H \phi_{0}}{\phi}<\breve{\eta}\left(t_{j_{i}}\right) \Longleftrightarrow \frac{H \phi_{0}}{\breve{\eta}\left(t_{j_{i}}\right)}<\phi \leq \frac{H \phi_{0}}{\breve{\eta}\left(t_{j_{i+1}}\right)} .
$$

Consequently, to express the inverse of $\breve{\eta}$ we define the characteristic functions

$$
\psi_{i}(\phi)= \begin{cases}1 & \text { if } \frac{H \phi_{0}}{\breve{\eta}\left(t_{j_{i}}\right)}<\phi \leq \frac{H \phi_{0}}{\breve{\eta}\left(t_{j_{i+1}}\right)}, \quad i=1, \ldots, n . \\ 0 \quad \text { otherwise, }\end{cases}
$$

Finally, (14) yields the following explicit formula for the estimated portion $\breve{f}_{\mathrm{b}}$ of $f_{\mathrm{b}}$ :

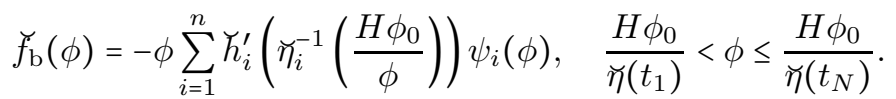

We note that $\breve{f}_{\mathrm{b}}$ has the same regularity as $\breve{h}$ by Theorem 2.1 .

3.2. Quadratic-fit method. A 3-parameter functional form with the quadratic polynomial $\breve{h}_{i}(t)=a_{i} t^{2}+b_{i} t+c_{i}$ together with (21), (22) yields that $\breve{h} \in C^{1}$. The convexity constraints (25) are $a_{i}>0$ for $i=1, \ldots, n-1$ and $a_{n} \geq 0$. We require now that all $a_{i}>0$. It is straightforward to compute

$$
\breve{\eta}_{i}(t)=-a_{i} t^{2}+c_{i} \text { and } \breve{\eta}_{i}^{-1}(y)=\sqrt{\frac{c_{i}-y}{a_{i}}},
$$

where we note that $a_{i}>0$ and $c_{i}>y$.

After the $3 n$ parameters have been determined (see Section 4), (27) yields

$$
\breve{f}_{\mathrm{b}}(\phi)=-\sum_{i=1}^{n}\left(b_{i} \phi+2 \sqrt{a_{i} \phi\left(c_{i} \phi-H \phi_{0}\right)}\right) \psi_{i}(\phi), \quad \frac{H \phi_{0}}{\breve{\eta}\left(t_{1}\right)}<\phi \leq \frac{H \phi_{0}}{\breve{\eta}\left(t_{N}\right)} .
$$

Here we note that $b_{i}<0$ since $a_{i}>0$ and $\breve{h}_{i}^{\prime}(t)=2 a_{i} t+b_{i}<0$, and that $\breve{f}_{\mathrm{b}} \in C^{1}$.

3.3. Spline-fit method. In order to increase the regularity so that $\breve{h}, \breve{f}_{\mathrm{b}} \in C^{2}$ one can use cubic polynomials $\breve{h}_{i}(t)=a_{i} t^{3}+b_{i} t^{2}+c_{i} t+d_{i}$. Then we have

$$
\breve{\eta}_{i}(t)=-2 a_{i} t^{3}-b_{i} t^{2}+d_{i}, \quad t \in \mathbb{R} .
$$

An explicit representation for the inverse $\breve{\eta}_{i}^{-1}$ requires some preparation. Note that the convexity conditions (25) are

$$
\begin{aligned}
\breve{h}_{i}^{\prime \prime}(t) & =2\left(3 a_{i} t+b_{i}\right)>0, \quad t_{j_{i}} \leq t<t_{j_{i+1}}, \quad i=1, \ldots, n-1, \\
\text { and } \quad \breve{h}_{n}^{\prime \prime}\left(t_{N}\right) & =2\left(3 a_{n} t_{N}+b_{n}\right) \geq 0,
\end{aligned}
$$


BÜRGER AND DIEHL

and that $\breve{\eta}_{i}^{\prime}(t)=-t \breve{h}_{i}^{\prime \prime}(t)=-2 t\left(3 a_{i} t+b_{i}\right)$, which should be negative in the interval $\left(t_{j_{i}}, t_{j_{i+1}}\right]$. Furthermore, $\breve{\eta}_{i}$ has the two stationary points $t=0$ and $t=-b_{i} /\left(3 a_{i}\right)$. In these points the function values are

$$
\breve{\eta}_{i}(0)=d_{i} \quad \text { and } \quad \breve{\eta}_{i}\left(-\frac{b_{i}}{3 a_{i}}\right)=d_{i}-\frac{b_{i}^{3}}{27 a_{i}^{2}} .
$$

To simplify calculations and implementation, we impose the additional constraints

$$
a_{i}<0 \quad \text { and } \quad b_{i}>0, \quad i=1, \ldots, n \text {. }
$$

(For the minimization problem, the more preferable constraints $b_{i} \geq 0$ could also have been used, but this leads to different explicit formulas for $\breve{\eta}_{i}^{-1}$ depending on whether $b_{i}=0$ or $b_{i}>0$ holds after the optimization.) Since $a_{i}<0$ if we impose (30), all functions $\breve{h}_{i}^{\prime \prime}$ are decreasing. This fact, together with the continuity constraints (23), implies that all inequalities (29) can be replaced by the single constraint

$$
\breve{h}_{n}^{\prime \prime}\left(t_{N}\right) \geq 0 \text {. }
$$

The graph of $\breve{\eta}_{i}$ has its decreasing part within the two stationary points, i.e. in the interval $\left(0,-b_{i} /\left(3 a_{i}\right)\right)$. Hence, when solving the equation

$$
\breve{\eta}_{i}(t)=y \quad \text { for a fixed } y \in\left(d_{i}-\frac{b_{i}^{3}}{27 a_{i}^{2}}, d_{i}\right),
$$

we are interested in the middle root of the three real ones. Details of the solution procedure can be found in the Appendix. The inverse is

$$
\breve{\eta}_{i}^{-1}(y)=-\frac{b_{i}}{6 a_{i}}\left(2 \cos \left(\frac{1}{3} \arccos \left(1-\frac{54\left(d_{i}-y\right) a_{i}^{2}}{b_{i}^{3}}\right)-\frac{2 \pi}{3}\right)+1\right) .
$$

This function should be used in formula (27) for the estimated flux function. The result is too long to write down and it is not advisable to use in a computer program, wherefore we instead summarize the method in Section 4.3.

3.4. Special-fit method. With the following four-parameter functional form we will obtain the same regularity as the splines:

$$
\breve{h}_{i}(t)=\frac{a_{i}}{t^{2}}+\frac{b_{i}}{t}+c_{i}+d_{i} t
$$

Then we have

$$
\begin{array}{ll}
\breve{h}_{i}^{\prime}(t)=-\frac{2 a_{i}}{t^{3}}-\frac{b_{i}}{t^{2}}+d_{i}, & \breve{\eta}_{i}(t)=\frac{3 a_{i}}{t^{2}}+\frac{2 b_{i}}{t}+c_{i} \\
\breve{h}_{i}^{\prime \prime}(t)=\frac{6 a_{i}}{t^{4}}+\frac{2 b_{i}}{t^{3}}, & \breve{\eta}_{i}^{\prime}(t)=-t \breve{h}_{i}^{\prime \prime}(t)=-\frac{6 a_{i}}{t^{3}}-\frac{2 b_{i}}{t^{2}} .
\end{array}
$$

The convexity conditions (25) are equivalent to

$$
\begin{aligned}
\breve{h}_{i}^{\prime \prime}(t) & =3 a_{i}+b_{i} t>0, \quad t_{j_{i}} \leq t<t_{j_{i+1}}, \quad i=1, \ldots, n-1, \\
\text { and } \quad \breve{h}_{n}^{\prime \prime}\left(t_{N}\right) & =3 a_{n} t_{N}+b_{n} \geq 0,
\end{aligned}
$$

which means that each $\breve{\eta}_{i}$ is decreasing, and, together with the continuity constraints (21) $-(22), \breve{\eta}$ is decreasing. By imposing the constraints

$$
a_{i}>0 \quad \text { and } \quad b_{i} \geq 0, \quad i=1, \ldots, n
$$


the convexity constraints (34) are satisfied. Hence, each $\breve{\eta}_{i}$ is invertible and the second-order equation $\breve{\eta}_{i}(t)=y$, that is,

$$
3 a_{i}+2 b_{i} t+\left(c_{i}-y\right) t^{2}=0
$$

has the unique positive solution

$$
t=\breve{\eta}_{i}^{-1}(y)=\frac{1}{y-c_{i}}\left(b_{i}+\sqrt{b_{i}^{2}+3 a_{i}\left(y-c_{i}\right)}\right) .
$$

Note that (35) and (36) imply that $y>c_{i}$. We get

$$
\breve{\eta}_{i}^{-1}\left(\frac{H \phi_{0}}{\phi}\right)=\frac{b_{i} \phi+\sqrt{\phi^{2}\left(b_{i}^{2}-3 a_{i} c_{i}\right)+3 a_{i} H \phi_{0} \phi}}{H \phi_{0}-c_{i} \phi},
$$

which should be composed with $\breve{h}_{i}^{\prime}$ in the formula (27) for the estimated flux function. This is too long to write down and it is not advisable to use in a computer program, wherefore we instead summarize the method in Section 4.3.

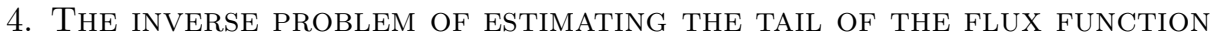 FROM A KYNCH TEST}

4.1. The quadratic-programming problem. To the given the $N$ data points (19), the parameters $a_{i}, b_{i}, c_{i}$ and $d_{i}$ should be determined as the unique solution of a linear least-squares minimization problem with constraints. We introduce the following vectors:

$$
\boldsymbol{p}_{i}:=\left(\begin{array}{c}
a_{i} \\
b_{i} \\
c_{i} \\
d_{i}
\end{array}\right), \quad \boldsymbol{p}:=\left(\begin{array}{c}
\boldsymbol{p}_{1} \\
\boldsymbol{p}_{2} \\
\vdots \\
\boldsymbol{p}_{n}
\end{array}\right), \quad \boldsymbol{x}_{i}:=\left(\begin{array}{c}
x_{j_{i}} \\
x_{j_{i}+1} \\
\vdots \\
x_{j_{i+1}-1}
\end{array}\right), \quad \boldsymbol{x}:=\left(\begin{array}{c}
\boldsymbol{x}_{1} \\
\boldsymbol{x}_{2} \\
\vdots \\
\boldsymbol{x}_{n} \\
x_{N}
\end{array}\right) .
$$

Then one can write $\breve{h}_{i}(t)=\boldsymbol{p}_{i}^{\mathrm{T}} \boldsymbol{q}(t)=\boldsymbol{q}(t)^{\mathrm{T}} \boldsymbol{p}_{i}$, where

$$
\boldsymbol{q}(t):=\left(\begin{array}{c}
t^{2} \\
t \\
1 \\
0
\end{array}\right), \quad\left(\begin{array}{c}
t^{3} \\
t^{2} \\
t \\
1
\end{array}\right), \quad \text { or } \quad\left(\begin{array}{c}
1 / t^{2} \\
1 / t \\
1 \\
t
\end{array}\right)
$$

to cover all our three fitting methods. Defining also the matrices

$$
\boldsymbol{Q}_{i}:=\left(\begin{array}{c}
\boldsymbol{q}\left(t_{j_{i}}\right)^{\mathrm{T}} \\
\boldsymbol{q}\left(t_{j_{i}+1}\right)^{\mathrm{T}} \\
\vdots \\
\boldsymbol{q}\left(t_{j_{i+1}-1}\right)^{\mathrm{T}}
\end{array}\right), \quad \boldsymbol{Q}:=\left(\begin{array}{cccc}
\boldsymbol{Q}_{1} & \mathbf{0} & \cdots & \mathbf{0} \\
\mathbf{0} & \boldsymbol{Q}_{2} & & \vdots \\
\vdots & & \ddots & \\
\mathbf{0} & \cdots & & \boldsymbol{Q}_{n} \\
\mathbf{0} & \cdots & & \boldsymbol{q}\left(t_{N}\right)^{\mathrm{T}}
\end{array}\right),
$$


we can express the objective function of the minimization problem as follows:

$$
\begin{aligned}
J(\boldsymbol{p}) & =\sum_{i=1}^{n} \sum_{k=j_{i}}^{j_{i+1}-1}\left(\breve{h}_{i}\left(t_{k}\right)-x_{k}\right)^{2}+\left(\breve{h}_{n}\left(t_{N}\right)-x_{N}\right)^{2} \\
& =\sum_{i=1}^{n} \sum_{k=j_{i}}^{j_{i+1}-1}\left(\boldsymbol{q}\left(t_{k}\right)^{\mathrm{T}} \boldsymbol{p}_{i}-x_{k}\right)^{2}+\left(\breve{h}_{n}\left(t_{N}\right)-x_{N}\right)^{2} \\
& =\sum_{i=1}^{n}\left(\boldsymbol{Q}_{i} \boldsymbol{p}_{i}-\boldsymbol{x}_{i}\right)^{\mathrm{T}}\left(\boldsymbol{Q}_{i} \boldsymbol{p}_{i}-\boldsymbol{x}_{i}\right)+\left(\breve{h}_{n}\left(t_{N}\right)-x_{N}\right)^{2} \\
& =(\boldsymbol{Q} \boldsymbol{p}-\boldsymbol{x})^{\mathrm{T}}(\boldsymbol{Q} \boldsymbol{p}-\boldsymbol{x})=\boldsymbol{p}^{\mathrm{T}} \boldsymbol{Q}^{\mathrm{T}} \boldsymbol{Q} \boldsymbol{p}-2 \boldsymbol{x}^{\mathrm{T}} \boldsymbol{Q} \boldsymbol{p}+\boldsymbol{x}^{\mathrm{T}} \boldsymbol{x} .
\end{aligned}
$$

We note that the size of $\boldsymbol{Q}$ is $N \times 4 n$ and the Hessian of $J$ has the size $4 n \times 4 n$.

To express the equality constraints (21)-(23) in standard matrix form, we define

$$
\begin{aligned}
\boldsymbol{R}_{i}^{\text {spline }}:=\boldsymbol{R}_{i}^{\text {spec }}:=\boldsymbol{R}_{i}:=\left(\begin{array}{c}
\boldsymbol{q}\left(t_{j_{i}}\right)^{\mathrm{T}} \\
\boldsymbol{q}^{\prime}\left(t_{j_{i}}\right)^{\mathrm{T}} \\
\boldsymbol{q}^{\prime \prime}\left(t_{j_{i}}\right)^{\mathrm{T}}
\end{array}\right), \\
\boldsymbol{R}^{\text {spline }}:=\boldsymbol{R}^{\text {spec }}:=\left(\begin{array}{ccccc}
\boldsymbol{R}_{2} & -\boldsymbol{R}_{2} & \mathbf{0} & \ldots & \mathbf{0} \\
\mathbf{0} & \boldsymbol{R}_{3} & -\boldsymbol{R}_{3} & \ldots & \mathbf{0} \\
\vdots & & \ddots & \ddots & \vdots \\
\mathbf{0} & \cdots & \mathbf{0} & \boldsymbol{R}_{n} & -\boldsymbol{R}_{n}
\end{array}\right) .
\end{aligned}
$$

For the quadratic-fit method, only (21)-(22) are used, wherefore we define

$$
\boldsymbol{R}_{i}^{\text {quad }}:=\left(\begin{array}{c}
\boldsymbol{q}\left(t_{j_{i}}\right)^{\mathrm{T}} \\
\boldsymbol{q}^{\prime}\left(t_{j_{i}}\right)^{\mathrm{T}}
\end{array}\right)
$$

and $\boldsymbol{R}^{\text {quad }}$ in the same ways as for the other methods. Then the equality constraints (21)-(23) are equivalent to $\boldsymbol{R}_{i}^{\text {method }}\left(\boldsymbol{p}_{i-1}-\boldsymbol{p}_{i}\right)=\mathbf{0}$ for $i=2, \ldots, n$, or more compactly, $\boldsymbol{R}^{\text {method }} \boldsymbol{p}=\mathbf{0}$, where "method" equals "quad", "spline" or "special".

The inequality constraints for the three methods are different and some are strict. Therefore, we let $\varepsilon>0$ be a small number and $\mathbf{1}_{m \times n}$ denote an $m \times n$ matrix full of ones. We define

$$
\begin{aligned}
& \boldsymbol{e}_{1}:=\left(\begin{array}{l}
1 \\
0 \\
0 \\
0
\end{array}\right), \quad \boldsymbol{e}_{2}:=\left(\begin{array}{l}
0 \\
1 \\
0 \\
0
\end{array}\right), \quad \boldsymbol{I}_{i}:=\left(\begin{array}{cccc}
\boldsymbol{e}_{i}^{\mathrm{T}} & \mathbf{0} & \cdots & \mathbf{0} \\
\mathbf{0} & \boldsymbol{e}_{i}^{\mathrm{T}} & & \vdots \\
\vdots & & \ddots & \mathbf{0} \\
\mathbf{0} & \cdots & \mathbf{0} & \boldsymbol{e}_{i}^{\mathrm{T}}
\end{array}\right)_{n \times(4 n)},
\end{aligned}
$$

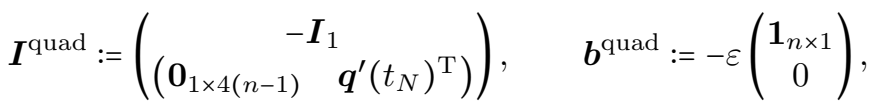

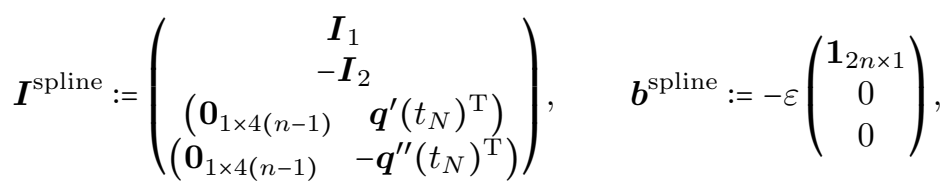

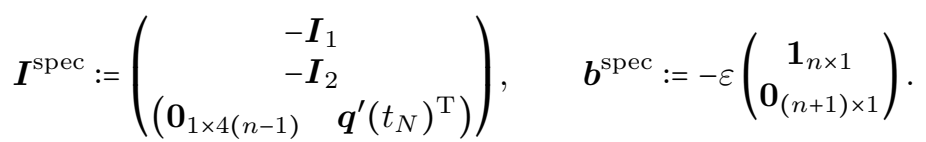

Then the inequality constraints $\boldsymbol{I}^{\text {method }} \boldsymbol{p} \leq \boldsymbol{b}^{\text {method }}$ imply (25) and (26). 
For data points (19) representing the curved discontinuity, the parameters $\boldsymbol{p}$ are determined by the following quadratic programming problem:

$$
\begin{array}{ll}
\text { minimize } & J(\boldsymbol{p})=(\boldsymbol{Q} \boldsymbol{p}-\boldsymbol{x})^{\mathrm{T}}(\boldsymbol{Q} \boldsymbol{p}-\boldsymbol{x}) \\
\text { subject to } & \boldsymbol{R}^{\text {method }} \boldsymbol{p}=\mathbf{0}, \quad \boldsymbol{I}^{\text {method }} \boldsymbol{p} \leq \boldsymbol{b}^{\text {method }},
\end{array}
$$

where "method" is equal to one of the three methods described above.

\subsection{Well-posedness of the inverse problem.}

Theorem 4.1. Consider the data points (19) and one of the three fitting methods described in Section 3. If the number of data points $N_{i}=j_{i+1}-j_{i}$ on each interval $\left(t_{j_{i}}, t_{j_{i+1}}\right]$ satisfies $N_{i} \geq p$, where $p$ is the number of parameters of $h_{i}$, then there exists a unique solution $\boldsymbol{p}$ of the quadratic program (40). Furthermore, the estimated tail of the flux function $\breve{f}_{\mathrm{b}}$, explicitly given by (14), depends continuously on the data points.

Proof. The uniqueness of the solution of (40) is obtained by proving that the Hes$\operatorname{sian} \nabla^{2} J=\boldsymbol{Q}^{\mathrm{T}} \boldsymbol{Q}$ is positive definite. To this end, we may temporarily consider the calculations in (38) with $\boldsymbol{x}=\mathbf{0}$ and let $\boldsymbol{p}$ be an arbitrary vector. Then $\boldsymbol{p}^{\mathrm{T}} \boldsymbol{Q}^{\mathrm{T}} \boldsymbol{Q} \boldsymbol{p} \geq 0$ holds, with equality if and only if

$$
\boldsymbol{q}\left(t_{k}\right)^{\mathrm{T}} \boldsymbol{p}_{i}=0, \quad k=j_{i}, \ldots, j_{i+1}-1, \quad i=1, \ldots, n, \quad \text { and } \quad \boldsymbol{q}\left(t_{N}\right)^{\mathrm{T}} \boldsymbol{p}_{n}=0 .
$$

These equalities are equivalent to the fact that each polynomial of degree $p-1$ has $N_{i} \geq p$ roots (the $t_{k}$ are distinct), which, in view of the fundamental theorem of algebra, implies that $\boldsymbol{p}=\mathbf{0}$. To prove that there exists a feasible solution of (40), we state explicitly a such for each of the methods. A feasible point for the quadratic-fit method is given by the following parameters:

$$
a_{i}=1, \quad b_{i}=-2 t_{N}, \quad c_{i}=t_{N}^{2}, \quad i=1, \ldots, n,
$$

which correspond to the (global) polynomial $\breve{h}(t)=\left(t-t_{N}\right)^{2}$. A feasible point for the spline-fit method is given by the following parameters:

$$
a_{i}=-1, \quad b_{i}=3 t_{N}, \quad c_{i}=-3 t_{N}^{2}, \quad d_{i}=t_{N}^{3}, \quad i=1, \ldots, n,
$$

which correspond to the (global) polynomial $\breve{h}(t)=-\left(t-t_{N}\right)^{3}$. A feasible point for the special-fit method is given by the following parameters:

$$
a_{i}=1, \quad b_{i}=c_{i}=0, \quad d_{i}=\frac{2}{t_{N}^{3}}, \quad i=1, \ldots, n,
$$

which correspond to the (global) function $\breve{h}(t)=1 / t^{2}+2 t / t_{N}^{3}$. It is straightforward to check that the constraints are satisfied in each of the three cases, and $\breve{f}_{\mathrm{b}}$ depends continuously on $\boldsymbol{p}$ since it is composed of functions that are continuous with respect $\boldsymbol{p}$. It is well known (cf. $[15,17])$ that the unique minimum $\boldsymbol{p}$ depends continuously on the matrices of the quadratic program (40), hence on the data points (19).

4.3. Summary of the three curve-fitting methods. As the coefficients $a_{i}, b_{i}$, $c_{i}, d_{i}$ have been determined as the unique solution of the quadratic programming problem (40), they should be plugged into the explicit formulas according to the following. For the quadratic-fit method, the expression for the tail of the batchsettling flux function is

$$
\breve{f}_{\mathrm{b}}(\phi)=-\sum_{i=1}^{n}\left(b_{i} \phi+2 \sqrt{a_{i} \phi\left(c_{i} \phi-H \phi_{0}\right)}\right) \psi_{i}(\phi), \quad \frac{H \phi_{0}}{\dddot{\eta}\left(t_{1}\right)}<\phi \leq \frac{H \phi_{0}}{\eta\left(t_{N}\right)} .
$$



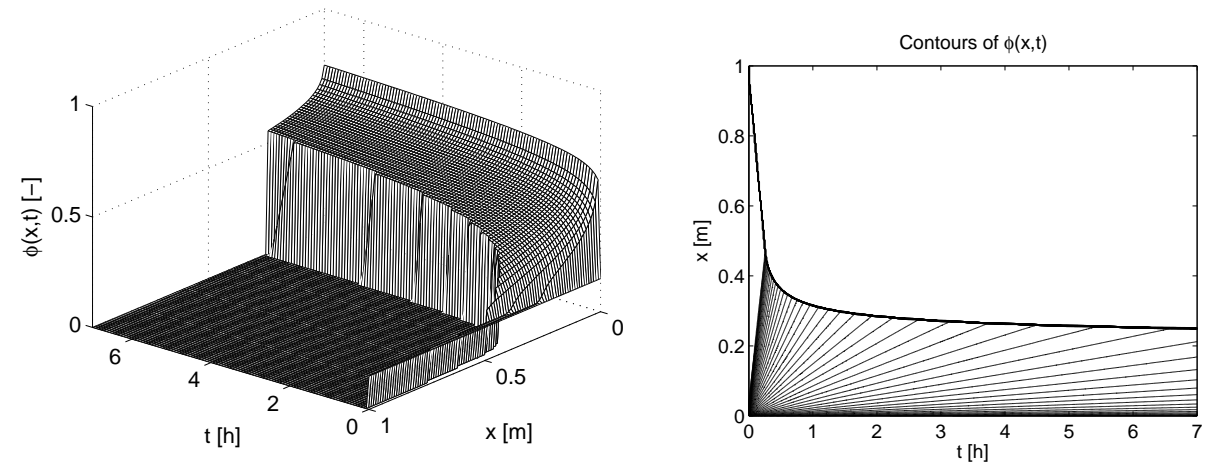

FiguRE 2. Three-dimensional graph (left) and contours (right) of the numerical solution of a Kynch test.

For the other two methods, the explicit expressions for $\breve{f}_{\mathrm{b}}$ are easier presented in the following step-wise ways. For each given volume fraction $\phi$ in the interval

$$
\frac{H \phi_{0}}{\breve{\eta}\left(t_{1}\right)}<\phi \leq \frac{H \phi_{0}}{\breve{\eta}\left(t_{N}\right)},
$$

one determines to which interval $\phi$ belongs; i.e., one determines the integer $I$ that satisfies

$$
\frac{H \phi_{0}}{\breve{\eta}\left(t_{j_{I}}\right)}<\phi \leq \frac{H \phi_{0}}{\breve{\eta}\left(t_{j_{I+1}}\right)}
$$

Then one sets $y:=H \phi_{0} / \phi$. For the spline-fit method one then computes

$$
\begin{aligned}
& \alpha_{I}:=\arccos \left(1-\frac{54 a_{I}^{2}\left(d_{I}-y\right)}{b_{I}^{3}}\right), \quad \tau_{I}:=-\frac{b_{I}}{6 a_{I}}\left(2 \cos \left(\frac{\alpha_{I}-2 \pi}{3}\right)+1\right), \\
& \breve{f}_{\mathrm{b}}(\phi):=-\phi \breve{h}_{I}^{\prime}\left(\tau_{I}\right) .
\end{aligned}
$$

and for the special-fit method, one computes

$$
\tau_{I}:=\frac{1}{y-c_{I}}\left(b_{I}+\sqrt{b_{I}^{2}+3 a_{I}\left(y-c_{I}\right)}\right), \quad \breve{\mathrm{b}}_{\mathrm{b}}(\phi):=-\phi \breve{h}_{I}^{\prime}\left(\tau_{I}\right) .
$$

4.4. Estimation from synthetic data. We exemplify the three estimation methods applied to synthetic data produced by a numerical simulation of a Kynch test, where the flux function is known. One advantage of using synthetic data is that the accuracies of the three methods can be compared. The simulation was performed with the Godunov numerical flux and the batch-settling flux function chosen is

$$
f_{\mathrm{b}}(\phi)=v_{0} \phi(1-\phi)^{n_{\mathrm{Rz}}},
$$

with $v_{0}=10$ and the Richardson-Zaki [52] exponent $n_{\mathrm{RZ}}=10$; see the dashed graph in Figure 3 (upper right). This function has an inflection point at $\phi_{\text {infl }}=2 / 11 \approx$ 0.182 . We have chosen an initial concentration slightly less than the inflection point; $\phi_{0}=0.15$. The numerical solution and contours of it are presented in Figure 2.

In (39) and (52), we have used $\varepsilon=10^{-5}$. The division of the data points into $n$ subintervals is made such that the points are evenly distributed with the remainder of the points put in the last interval. From the contour plot 47 data points were selected on the computer screen. Some estimation results of the three methods are 

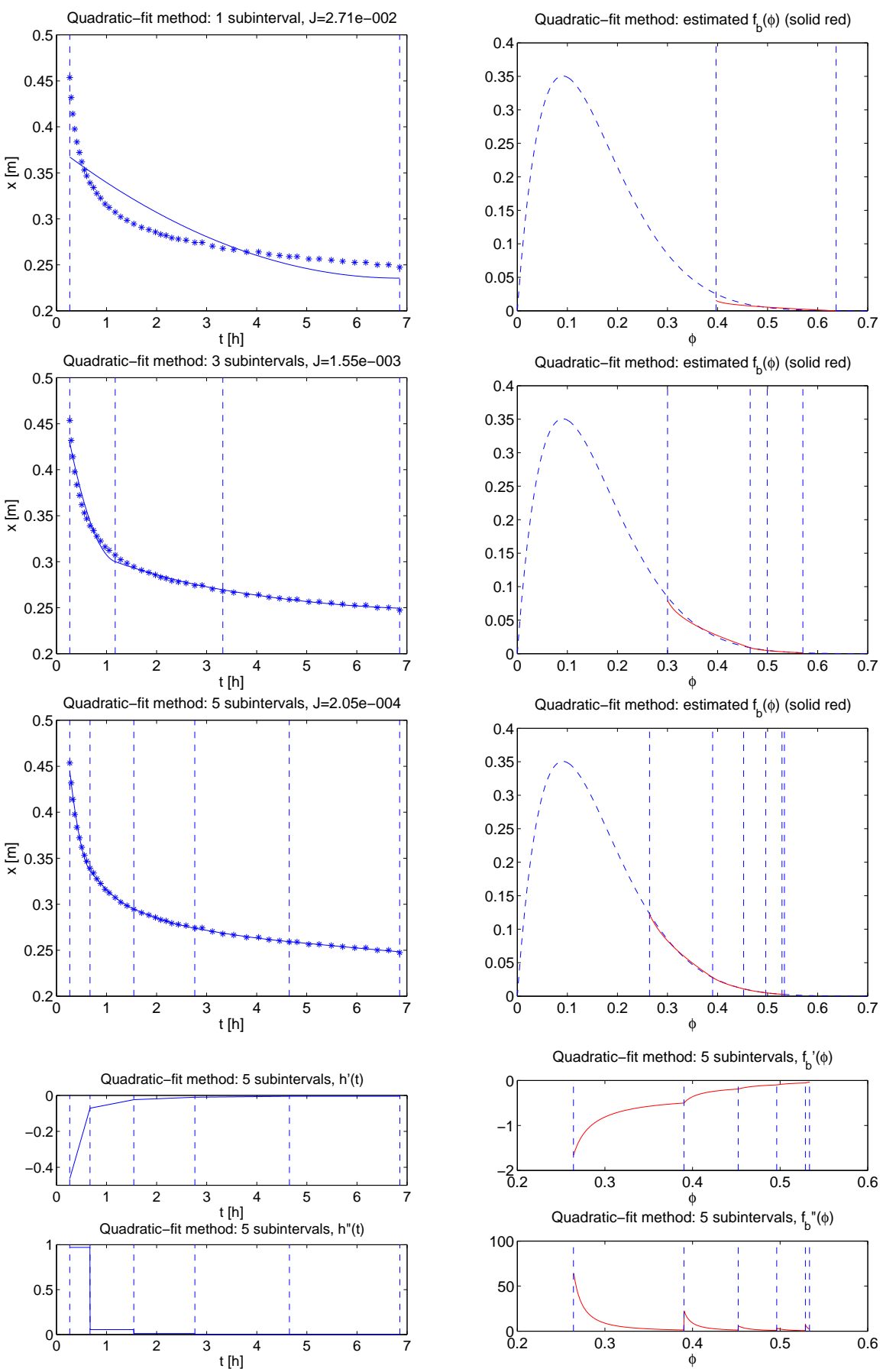

FiguRE 3. Quadratic-fit method. The value of $J$ is the minimum for the estimated $\breve{h}$. Note that the estimated $\breve{h}, \breve{f}_{\mathrm{b}} \in C^{1} \backslash C^{2}$.

shown in Figures $3-5$. Note that the estimated functions $\breve{h}$ and $\breve{\mathrm{b}_{\mathrm{b}}}$ have the same 

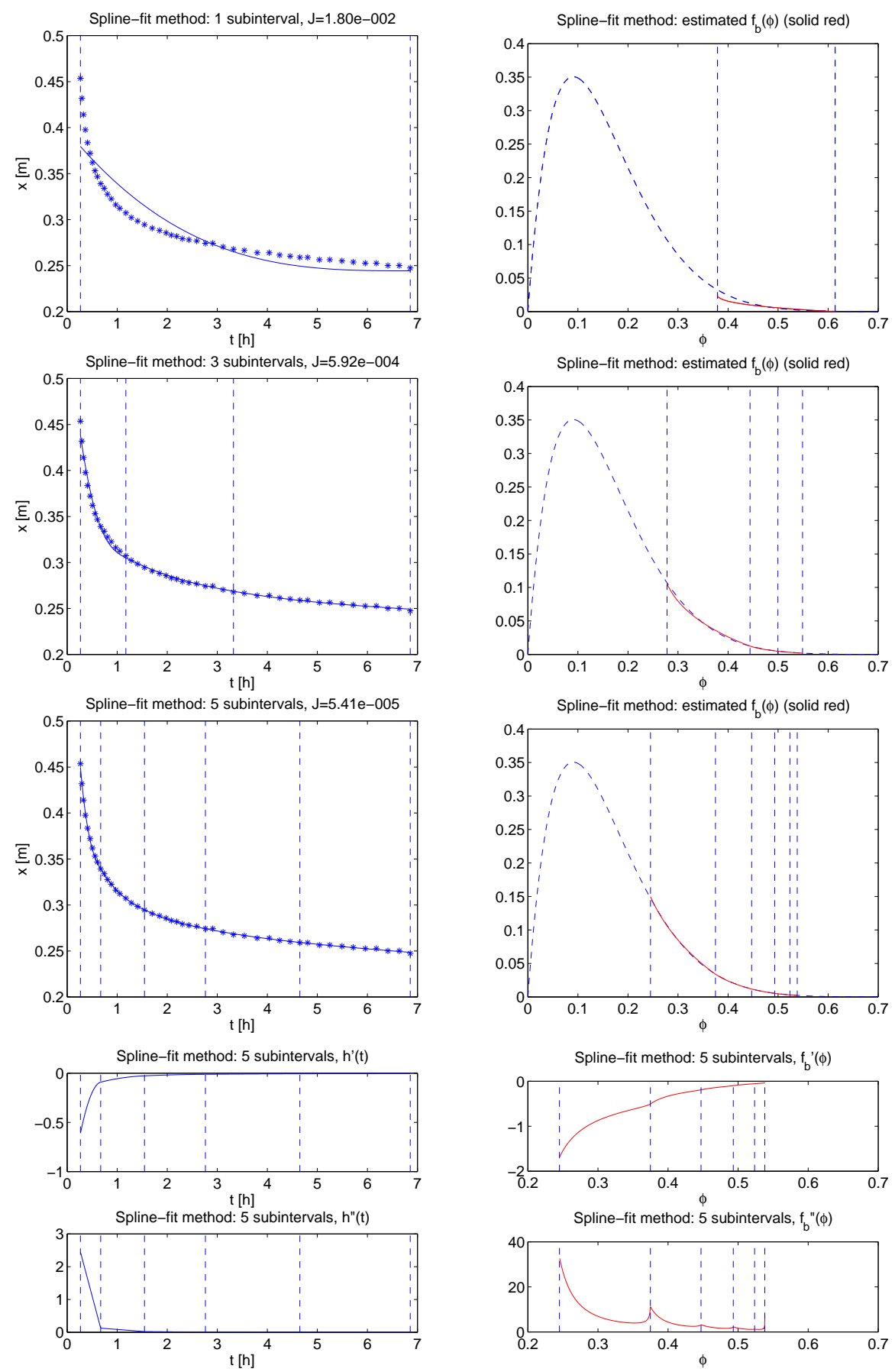

Figure 4. Spline-fit method for the estimation of $\breve{h}, \breve{\mathrm{b}} \in C^{2}$.

regularity. For the special-fit method, there seems to be no improvement with more than one subinterval; see Figures 5 and 6 . 

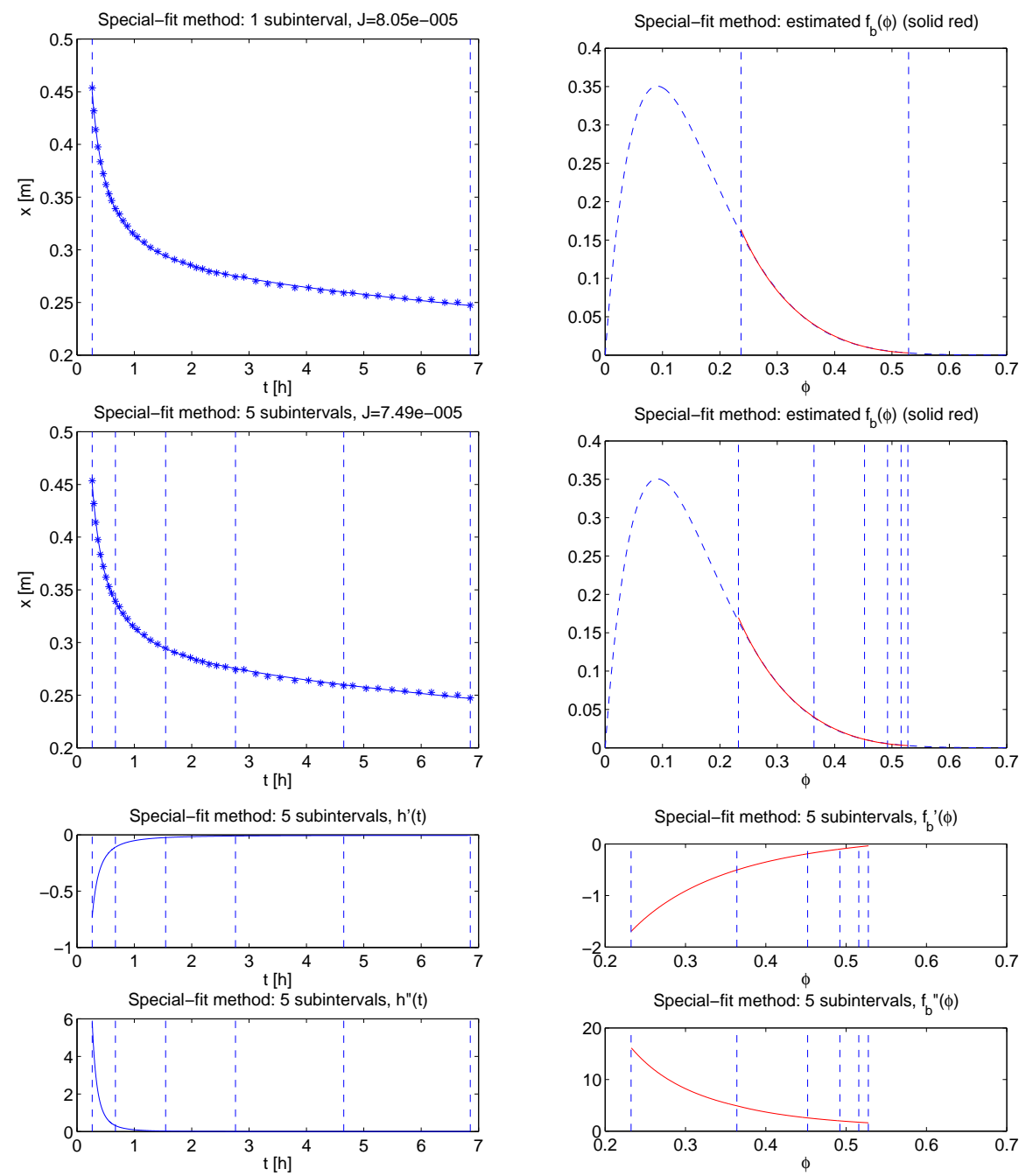

FIGURE 5. Special-fit method with $\breve{h}, \breve{b}_{\mathrm{b}} \in C^{2}$.

\section{The inVerse Problem of estimating a CONCAVE PART of the FluX FUNCTION FROM A DIEHL TEST}

The initial data are now

$$
\phi_{\text {init }}(x)= \begin{cases}0 & \text { for } 0<x<H_{0}, \\ \phi_{0} & \text { for } H_{0} \leq x<H,\end{cases}
$$

where $H_{0}$ is the height of clear liquid from the bottom. The solution of (2) with the initial data (42) and $\phi_{\text {infl }}<\phi_{0}<\phi_{\max }$ is shown in Figure 7. For details on the solution, including the cases not treated here, we refer to [21]. (In the case $\phi_{0}=\phi_{\max }$, the uppermost first discontinuity has zero speed, i.e., it stays at the liquid surface until $t=\hat{t}_{\text {start }}$.) Of our interest is the upper curved discontinuity $x=h(t)$ for $\hat{t}_{\text {start }}<t<\hat{t}_{\text {end }}$, which is now concave. Below this, the concentration 


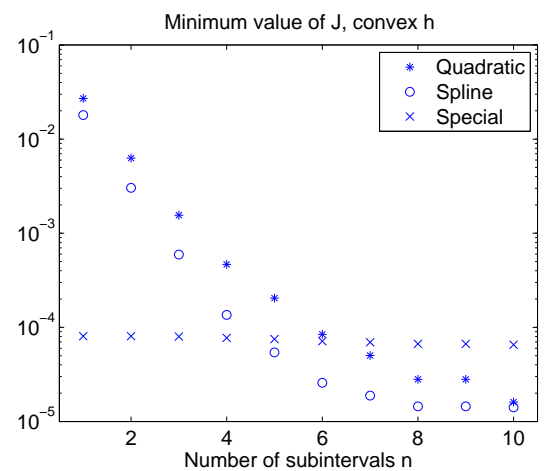

Figure 6. Values of $J$ in the minima of the quadratic program (40) for different number of subintervals for $\breve{h}$.
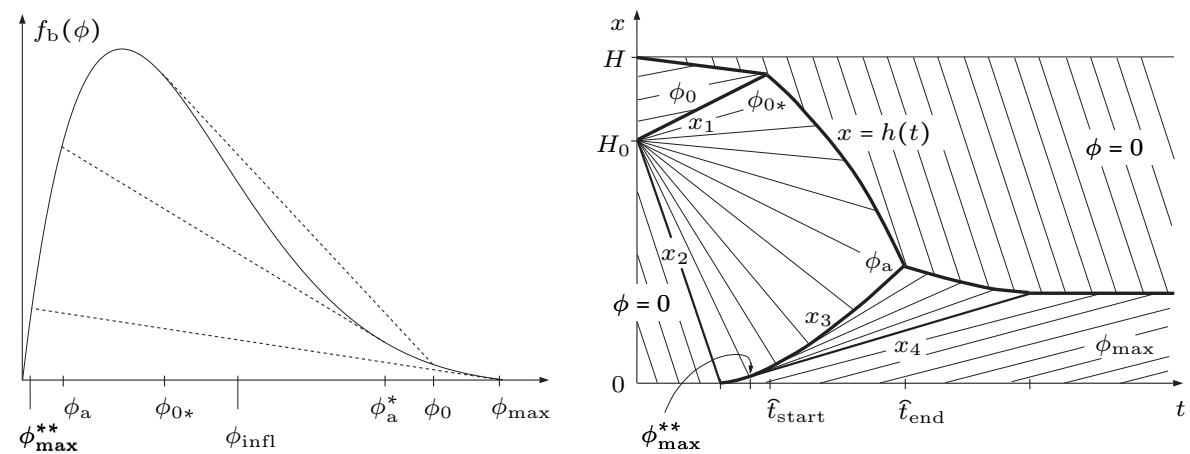

Figure 7. Left: Flux function with some of the concentrations appearing in the solution. Right: Schematic solution of the Diehl test with zero concentration below $x=H_{0}$ and a uniform concentration $\phi_{0}$ above. The interval of estimation is $\left[\phi_{\mathrm{a}}, \phi_{0 *}\right](\mathrm{cf} .(5))$.

values, in the expansion wave originating at $\left(0, H_{0}\right)$, decreases from $\phi_{0 *}$ to $\phi_{\mathrm{a}}$, where the latter is unknown beforehand but can be calculated given the time point $t_{\mathrm{end}}$ :

$$
\phi_{\mathrm{a}}=\frac{\left(H-H_{0}\right) \phi_{0}}{\eta\left(\hat{t}_{\text {start }}\right)-H_{0}} .
$$

Note that the interval $\left[\phi_{\mathrm{a}}, \phi_{0 *}\right]$ corresponds to a concave part of the flux function $f_{\mathrm{b}}$.

With similar derivations as for the convex tail of $f_{\mathrm{b}}$ and the convex $h$ of the Kynch test, one can obtain the parametric formulas for the concave part of $f_{\mathrm{b}}$ :

$$
\left\{\begin{array}{rl}
\phi & =\frac{\left(H-H_{0}\right) \phi_{0}}{\eta(t)-H_{0}}, \\
f_{\mathrm{b}}(\phi) & =-\frac{\left(H-H_{0}\right) \phi_{0}}{\eta(t)-H_{0}} h^{\prime}(t),
\end{array} \quad \hat{t}_{\mathrm{start}} \leq t \leq \hat{t}_{\mathrm{end}},\right.
$$


where $\eta(t)=h(t)-t h^{\prime}(t)$ as before. The explicit representation is

$$
f_{\mathrm{b}}(\phi)=-\phi h^{\prime}\left(\eta^{-1}\left(H_{0}+\frac{\left(H-H_{0}\right) \phi_{0}}{\phi}\right)\right), \quad \phi_{\mathrm{a}} \leq \phi \leq \phi_{0 *},
$$

where $\phi_{\mathrm{a}}$ is given by (43). By analogy with the analysis in Section 2.3, one can conclude that $h, f_{\mathrm{b}} \in C^{2}$ are strictly concave functions and that $\eta$ is increasing. Furthermore, the analogous results of Lemma 2.1 and Theorem 2.1 hold, so that $\widehat{h}$ and $\hat{f}_{\mathrm{b}}$ have the same regularity.

Given $M$ data points, a piecewise fitting of $m$ functional forms can be done in a similar way as in Section 3, however, with some adjustments. For the estimated functions corresponding to the concave part of the flux, we use the accent $\sim$ instead of $\smile$. Inequalities (24) and (25) are replaced by

$$
\begin{aligned}
& \widehat{h}_{i}^{\prime}(t)<0, \quad t_{j_{i}} \leq t \leq t_{j_{i+1}}, \quad i=1, \ldots, m, \\
& \widehat{h}_{i}^{\prime \prime}(t)<0, \quad t_{j_{i}} \leq t \leq t_{j_{i+1}}, \quad i=1, \ldots, m,
\end{aligned}
$$

Since $\hat{h}^{\prime}$ is now decreasing, all inequalities in (44) can be replaced by the single inequality $\hat{h}_{1}^{\prime}\left(t_{1}\right)<0$. This replaces $(26)$ for the Kynch test. Most of the other inequality constraints are now reversed (in comparison with Section 3 ).

The explicit formulas for the estimated $\widehat{f}_{\mathrm{b}}$ can be obtained in the following ways. Consider for simplicity a given subinterval of $\left[0, \phi_{\max }\right]$ :

$$
\left(\frac{\left(H-H_{0}\right) \phi_{0}}{\eta\left(t_{j_{i+1}}\right)-H_{0}}, \frac{\left(H-H_{0}\right) \phi_{0}}{\eta\left(t_{j_{i}}\right)-H_{0}}\right] .
$$

For the quadratic-fit method, we require that all $a_{i}<0$ (reversed in comparison to the Kynch test). The inverse of $\eta$ is the same as for $\breve{\eta}$, but the explicit formula for the subinterval (46) is slightly different:

$$
\widehat{f}_{\mathrm{b}}(\phi)=2 \sqrt{a_{i} \phi\left(\left(c_{i}-H_{0}\right) \phi-\left(H-H_{0}\right) \phi_{0}\right)}-b_{i} \phi,
$$

For the spline-fit method, we require that the coefficients have the opposite signs as for the Kynch test, i.e., $a_{i}>0$ and $b_{i}<0$. Then $\eta$ has the same two stationary points as in the Kynch test but is now increasing in the interval between these; $\left(0,-b_{i} /\left(3 a_{i}\right)\right)$. Hence, when solving the equation

$$
\hat{\eta}_{i}(t)=y \quad \text { for a fixed } y \in\left(d_{i}, d_{i}-\frac{b_{i}^{3}}{27 a_{i}^{2}}\right),
$$

we are again interested in the middle root of the three real ones. Note that $d_{i}<y$ (opposite to the Kynch test). The inversion of the cubic polynomial in the Appendix can be repeated with the conclusion that $p<0$ and $|q|<-b^{3} /\left(108 a^{3}\right)$, which are the same as for the Kynch test. Hence, the same final formula is valid, i.e., set

$$
y:=H_{0}+\frac{\left(H-H_{0}\right) \phi_{0}}{\phi},
$$

and calculate

$$
\begin{aligned}
& \alpha_{i}:=\arccos \left(1-\frac{54 a_{i}^{2}\left(d_{i}-y\right)}{b_{i}^{3}}\right), \quad \tau_{i}:=-\frac{b_{i}}{6 a_{i}}\left(2 \cos \left(\frac{\alpha_{i}-2 \pi}{3}\right)+1\right), \\
& \widehat{f}_{\mathrm{b}}(\phi):=-\phi \hat{h}_{i}^{\prime}\left(\tau_{i}\right) .
\end{aligned}
$$


For the special-fit method, we require $a_{i}<0$ and $b_{i} \leq 0$. Then the unique positive inverse of $\hat{\eta}$ is

$$
\eta_{i}^{-1}(y)=\frac{1}{c_{i}-y}\left(-b_{i}+\sqrt{b_{i}^{2}+3 a_{i}\left(y-c_{i}\right)}\right) .
$$

(with $c_{i}>y$ ) instead of (37) for the Kynch test. Inserting (49), one obtains

$$
\begin{aligned}
\tau_{i}(\phi) & :=\widehat{\eta}_{i}^{-1}\left(H_{0}+\frac{\left(H-H_{0}\right) \phi_{0}}{\phi}\right) \\
& =\frac{-b_{i} \phi+\sqrt{\phi^{2}\left(b_{i}^{2}+3 a_{i}\left(H_{0}-c_{i}\right)\right)+\phi 3 a_{i} \phi_{0}\left(H-H_{0}\right)}}{\phi\left(H_{0}-c_{i}\right)+\phi_{0}\left(H-H_{0}\right)} .
\end{aligned}
$$

Finally, set $\hat{f}_{\mathrm{b}}(\phi):=-\phi \hat{h}_{i}^{\prime}\left(\tau_{i}(\phi)\right)$.

For the quadratic program (40), the inequality constraints are changed. All coefficient inequalities are reversed and (44) holds. Furthermore, for the spline-fit method, $a_{i}>0$ and $b_{i}<0$ imply that $\widehat{h}_{i}^{\prime \prime}(t)=2\left(3 a_{i} t+b_{i}\right)$ is increasing. Hence, all inequalities in (45) are satisfied if $\widehat{h}_{m}^{\prime \prime}\left(t_{M}\right)<0$. This inequality replaces (31) for the Kynch test. Therefore, the matrices

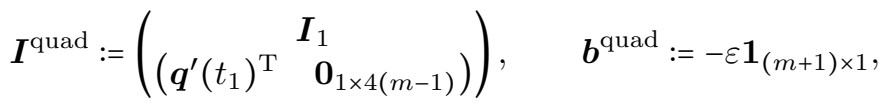

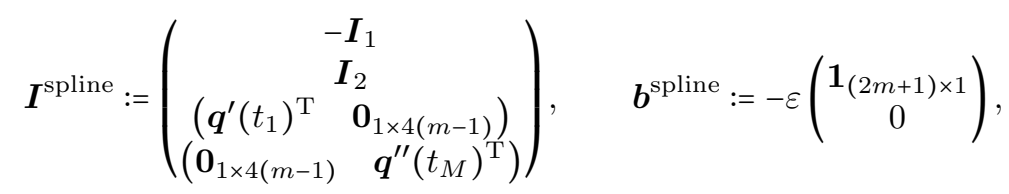

$$
\begin{aligned}
& \boldsymbol{I}^{\text {spec }}:=\left(\begin{array}{c}
\boldsymbol{I}_{1} \\
\boldsymbol{I}_{2} \\
\left(\boldsymbol{q}^{\prime}\left(t_{1}\right)^{\mathrm{T}}\right. \\
\left.\mathbf{0}_{1 \times 4(m-1)}\right)
\end{array}\right), \quad \boldsymbol{b}^{\text {spec }}:=-\varepsilon\left(\begin{array}{c}
\mathbf{1}_{m \times 1} \\
\mathbf{0}_{m \times 1} \\
1
\end{array}\right)
\end{aligned}
$$

are used in the optimization problem

$$
\begin{array}{ll}
\text { minimize } & J(\boldsymbol{p})=(\boldsymbol{Q} \boldsymbol{p}-\boldsymbol{x})^{\mathrm{T}}(\boldsymbol{Q} \boldsymbol{p}-\boldsymbol{x}) \\
\text { subject to } & \boldsymbol{R}^{\text {method }} \boldsymbol{p}=\mathbf{0}, \quad \boldsymbol{I}^{\text {method }} \boldsymbol{p} \leq \boldsymbol{b}^{\text {method }}
\end{array}
$$

where "method" is equal to one of the three methods. We finally note that a corresponding well-posedness result to Theorem 4.1 also holds for the case of determining $\hat{f}_{\mathrm{b}}$.

5.1. Estimation from synthetic data. By analogy with the examples in Section 4.4 for the Kynch test, we here show estimations from synthetic data for the Diehl test produced by simulations with the flux function (41). The (upper) initial concentration is slightly larger than the inflection point $\left(\phi_{\text {infl }}=2 / 11 \approx 0.182\right)$; $\phi_{0}=0.25$. The results of the simulation can be found in Figure 8 .

From the contour plot 37 data points were selected on the computer screen. Some results of the three methods are presented in Figures 9-11.

We note that the special-fit method is not able to produce an acceptable estimation in this example. Finally, we show in Figure 12 the values of $J$ of the minimization problem. 

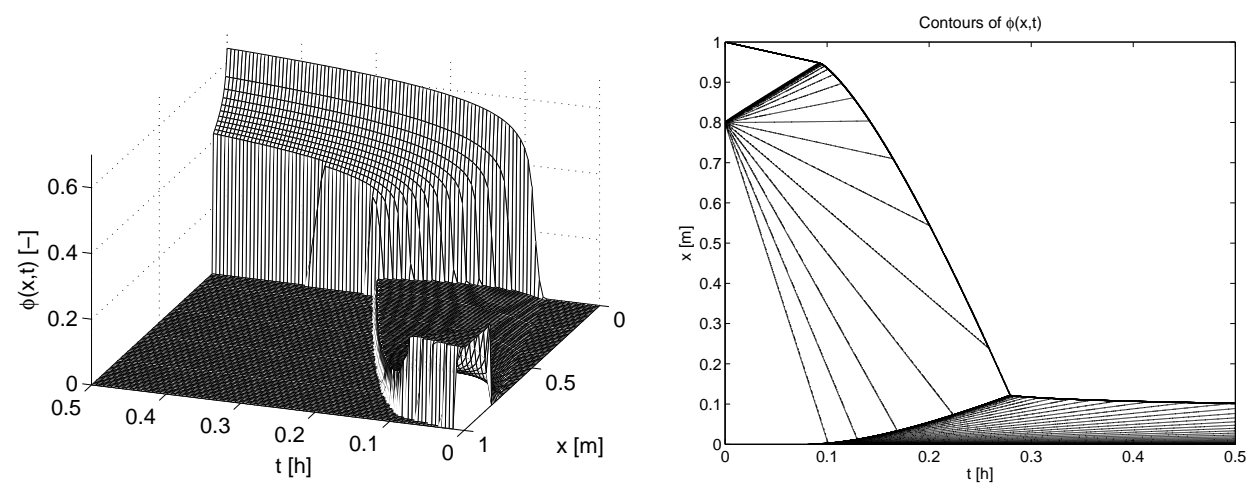

FiguRE 8. Three-dimensional graph (left) and contours (right) of the numerical solution of the Diehl test.

\section{Obtaining the Entire Flux FUnCtion}

To obtain an entire estimated flux function, denoted by $\bar{f}_{\mathrm{b}}$, the estimated convex and concave parts have to be complemented in the following three intervals of $\phi$ :

$$
\begin{array}{ll}
\text { Left: } & 0 \leq \phi \leq \frac{\left(H-H_{0}\right) \phi_{0}}{\eta\left(t_{M}\right)-H_{0}}=: \phi_{1}, \\
\text { Middle: } & \phi_{2}:=\frac{\left(H-H_{0}\right) \phi_{0}}{\eta\left(t_{1}\right)-H_{0}}<\phi<\frac{H \phi_{0}}{\breve{\eta}\left(t_{1}\right)}=: \phi_{3}, \\
\text { Right: } & \phi_{4}:=\frac{H \phi_{0}}{\eta\left(t_{M}\right)} \leq \phi \leq \phi_{\max } .
\end{array}
$$

6.1. The left interval. For the left interval $\left[0, \phi_{1}\right]$, one may extend the leftmost estimated function for the concave part

$$
\widehat{f}_{\mathrm{b}}(\phi)=-\phi \widehat{h}_{m}^{\prime}\left(\hat{\eta}_{m}^{-1}\left(H_{0}+\frac{\left(H-H_{0}\right) \phi_{0}}{\phi}\right)\right), \quad \phi_{1} \leq \phi \leq \frac{\left(H-H_{0}\right) \phi_{0}}{\widehat{\eta}\left(t_{M-1}\right)-H_{0}}
$$

to the larger interval

$$
0 \leq \phi \leq \frac{\left(H-H_{0}\right) \phi_{0}}{\eta\left(t_{M-1}\right)-H_{0}} .
$$

It is essential that $\hat{f}_{\mathrm{b}}(\phi) \searrow 0$ as $\phi \searrow 0$. This holds for the quadratic-fit method, which follows from the explicit formula (47). For the special-fit method, (51) yields

$$
\lim _{\phi \searrow 0} \tau_{m}(\phi)=\lim _{\phi \searrow 0} \hat{\eta}_{m}^{-1}\left(H_{0}+\frac{\left(H-H_{0}\right) \phi_{0}}{\phi}\right)=0 .
$$

Hence

$$
\widehat{f}_{\mathrm{b}}(0)=-\lim _{\phi \searrow 0} \phi \widehat{h}_{m}^{\prime}\left(\tau_{m}(\phi)\right)=0
$$

For the spline-fit method, this cannot be done since $\phi \searrow 0$ implies $y \rightarrow \infty$ in (49) and then $\alpha_{i}$ in (50) is not defined. Instead, one can fit a quadratic polynomial $r_{01}$ to define $\bar{f}_{\mathrm{b}}$ all the way down to $\phi=0$. The unique such polynomial that 

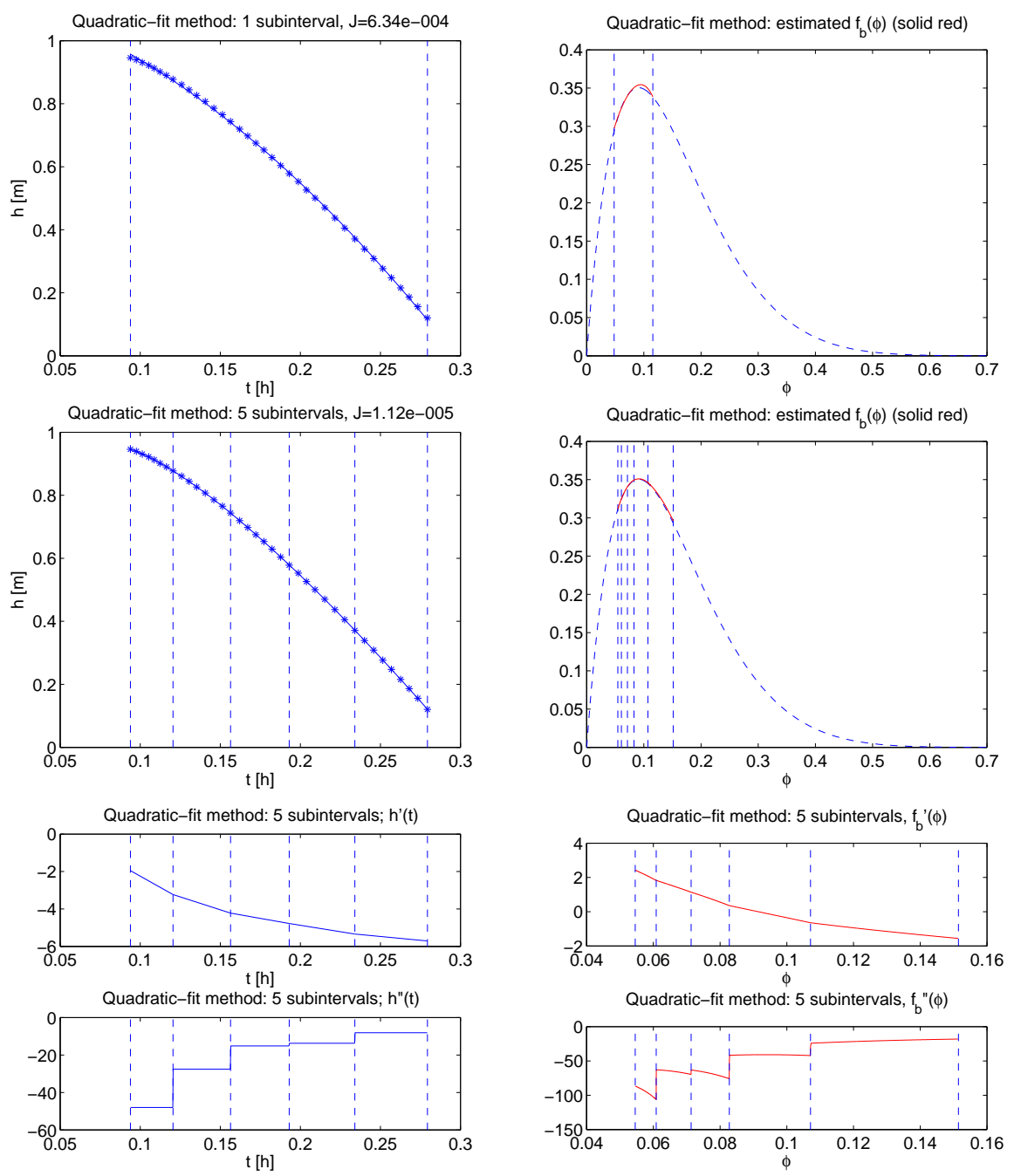

Figure 9. Quadratic-fit method with 1 (two uppermost plots) and 5 intervals for the estimation of $\widehat{h}$.

satisfies $r_{01}(0)=0, r_{01}\left(\phi_{1}\right)=\hat{f}_{\mathrm{b}}\left(\phi_{1}\right)$ and $r_{01}^{\prime}\left(\phi_{1}\right)=\hat{f}_{\mathrm{b}}^{\prime}\left(\phi_{1}\right)$ is given by the following expression, where $\Phi$ is defined by (11):

$$
r_{01}(\phi)=\frac{\phi}{\phi_{1}^{2}}\left(\phi_{1} \widehat{f}_{\mathrm{b}}\left(\phi_{1}\right)-\Phi\left(\phi_{1}\right)\left(\phi-\phi_{1}\right)\right) .
$$

This means that $\bar{f}_{\mathrm{b}} \in C^{1}$ at $\phi_{1}$. The numerical value of $\hat{f}_{\mathrm{b}}^{\prime}\left(\phi_{1}\right)$ can, in theory, be obtained easily by the corresponding formula of (8) for the Diehl test (see [21, Eq. $(27)]) ; \widehat{f}_{\mathrm{b}}^{\prime}\left(\phi_{1}\right)=\widehat{f}_{\mathrm{b}}^{\prime}\left(\phi_{h}\left(t_{\text {end }}\right)\right)=-\left(h\left(t_{\text {end }}\right)-H_{0}\right) / t_{\text {end }}$. Because of error in the data, this is not recommended. Differentiation of the explicitly obtained expression $\hat{f}_{\mathrm{b}}$ is of course possible, but a finite difference calculation (using $\widehat{f}_{\mathrm{b}}$ ) is easiest.

6.2. The middle interval. Due to various types of error, one may have $\phi_{2} \geq \phi_{3}$ after the optimization problem is solved, so that (54) are false. To proceed, we 

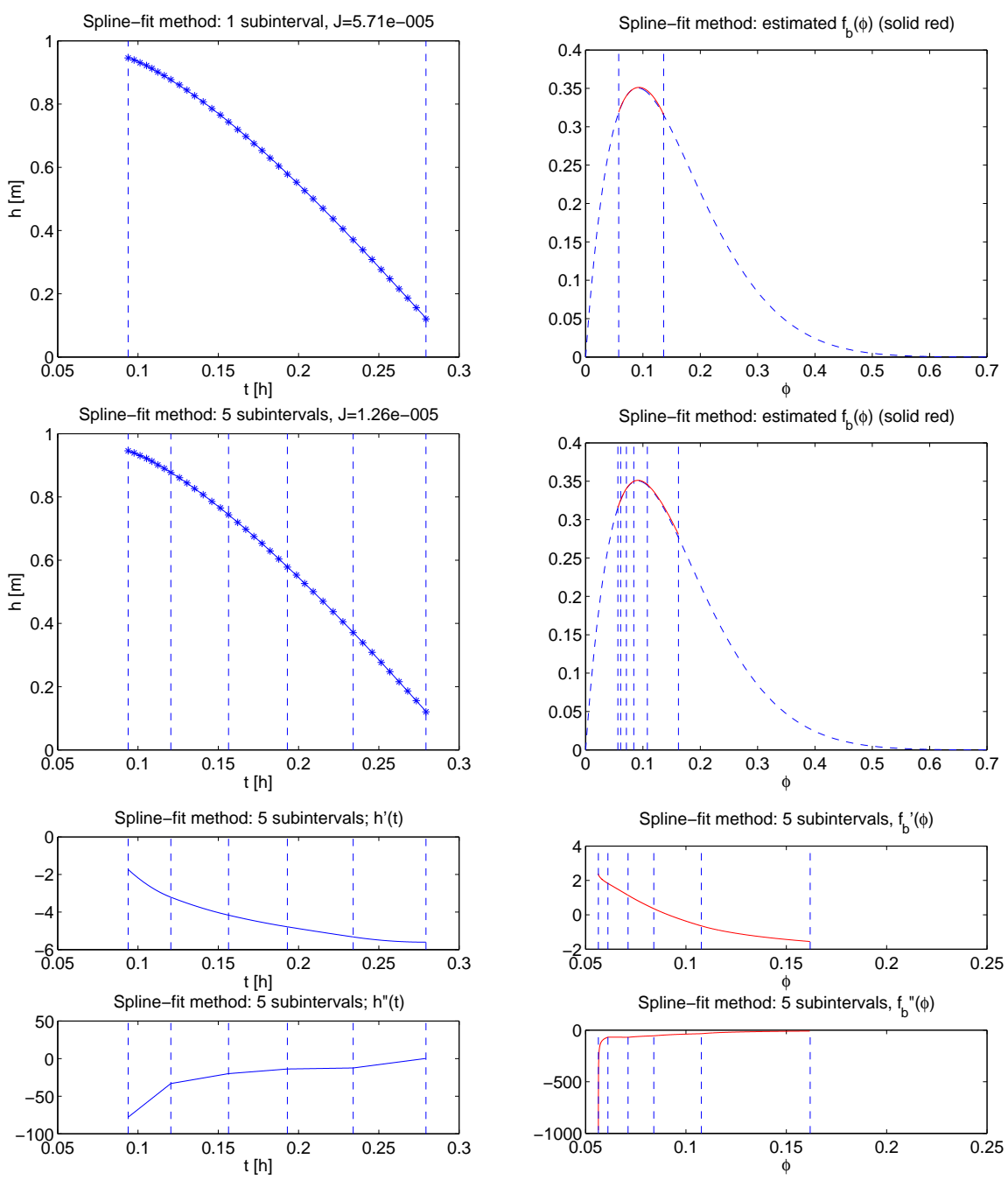

Figure 10. Spline-fit method with 1 (two uppermost plots) and 5 intervals for the estimation of $\hat{h}$.

simply assume that the interval $\left(\phi_{2}, \phi_{3}\right)$ has a positive length. In this interval, one can fit a cubic polynomial

$$
r_{23}(\phi):=a_{23}\left(\phi-\phi_{2}\right)\left(\phi-\phi_{3}\right)^{2}+b_{23}\left(\phi-\phi_{2}\right)\left(\phi-\phi_{3}\right)+c_{23}\left(\phi-\phi_{2}\right)+d_{23}
$$

satisfying the four equations

$$
r_{23}\left(\phi_{2}\right)=\widehat{f}_{\mathrm{b}}\left(\phi_{2}\right), \quad r_{23}^{\prime}\left(\phi_{2}\right)=\widehat{f}_{\mathrm{b}}^{\prime}\left(\phi_{2}\right), \quad r_{23}\left(\phi_{3}\right)=\breve{f}_{\mathrm{b}}\left(\phi_{3}\right), \quad r_{23}^{\prime}\left(\phi_{3}\right)=\breve{f}_{\mathrm{b}}^{\prime}\left(\phi_{3}\right),
$$

which have a unique solution when $\phi_{2} \neq \phi_{3}$. The coefficients are then given by

$$
a_{23}=\frac{2\left(\widehat{f}_{\mathrm{b}}\left(\phi_{3}\right)-\breve{f}_{\mathrm{b}}\left(\phi_{2}\right)\right)+\left(\phi_{2}-\phi_{3}\right)\left(\breve{f}_{\mathrm{b}}^{\prime}\left(\phi_{2}\right)+\widehat{f}_{\mathrm{b}}{ }^{\prime}\left(\phi_{3}\right)\right)}{\left(\phi_{2}-\phi_{3}\right)^{3}},
$$



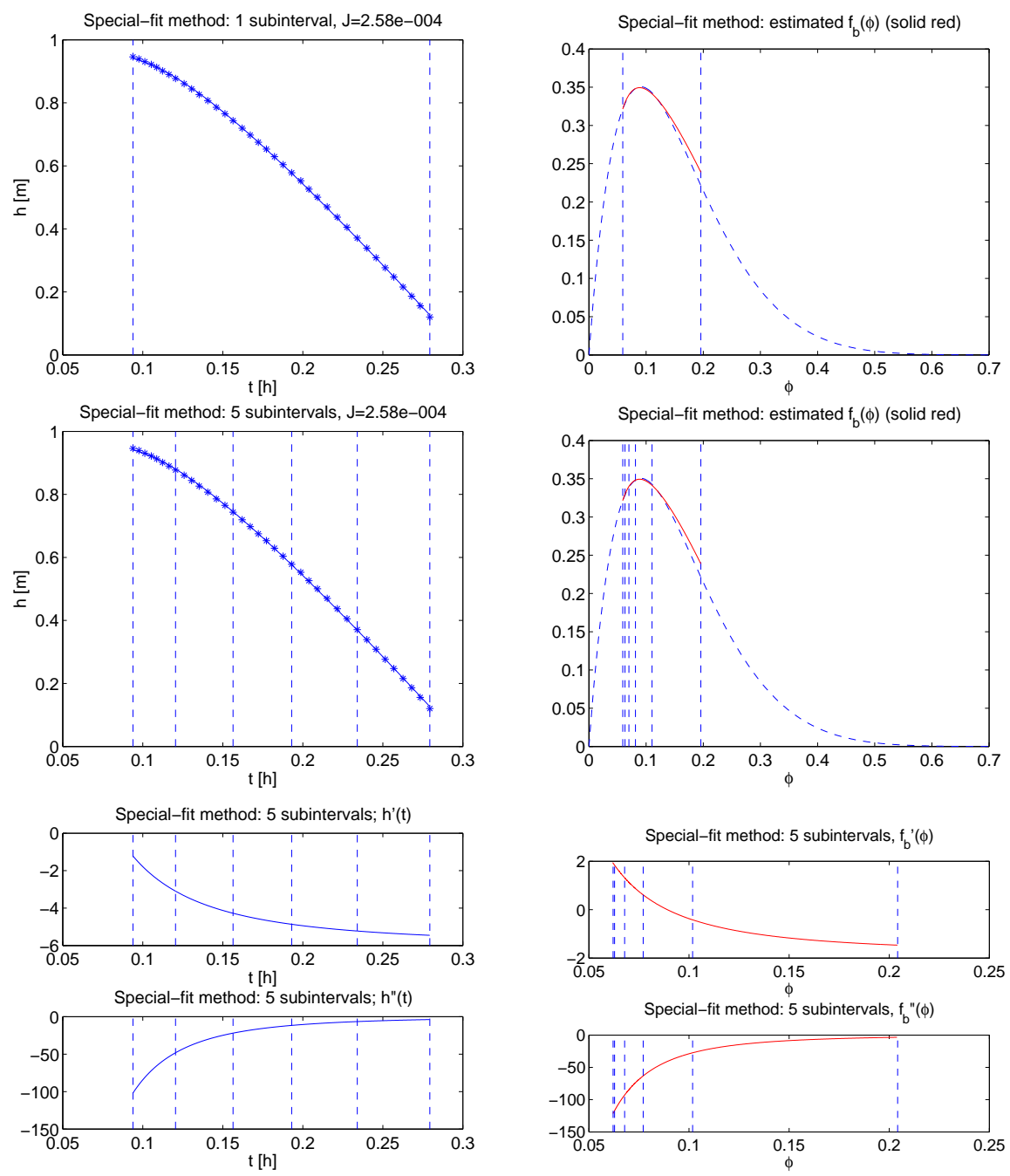

FIGURE 11. Special-fit method with 1 (two uppermost plots) and 5 intervals for the estimation of $\widehat{h}$.

$$
\begin{aligned}
b_{23} & =\frac{\breve{f}_{\mathrm{b}}\left(\phi_{2}\right)-\widehat{f}_{\mathrm{b}}\left(\phi_{3}\right)+\phi_{3} \breve{f}_{\mathrm{b}}^{\prime}\left(\phi_{2}\right)-\phi_{2} \widehat{f}_{\mathrm{b}}{ }^{\prime}\left(\phi_{3}\right)}{\left(\phi_{2}-\phi_{3}\right)^{2}}, \\
c_{23} & =\frac{\breve{f}_{\mathrm{b}}\left(\phi_{2}\right)-\widehat{f}_{\mathrm{b}}\left(\phi_{3}\right)}{\phi_{2}-\phi_{3}}, \quad d_{23}=\breve{f}_{\mathrm{b}}\left(\phi_{2}\right) .
\end{aligned}
$$

This means that $\bar{f}_{\mathrm{b}} \in C^{1}$ at $\phi_{2}$ and $\phi_{3}$. Now, one should check whether $f_{\mathrm{b}}$ has precisely one inflection point $\phi_{\text {inf }} \in\left[\phi_{2}, \phi_{3}\right]$ with $r_{23}^{\prime \prime \prime}\left(\phi_{\text {infl }}\right)>0$. Generically, the numerical values of the coefficients $a_{23}, \ldots, d_{23}$ are nonzero; however, we may have the following cases, where the last case is the preferred one:

- If $a_{23}=0$ and $b_{23}=0$, then $r_{23}$ is linear and $\bar{f}_{\mathrm{b}}^{\prime \prime}(\phi)=0$ for $\phi \in\left(\phi_{2}, \phi_{3}\right)$.

- If $a_{23}=0$ and $b_{23} \neq 0$, then $r_{23}$ is a quadratic function. If $r_{23}^{\prime \prime}\left(\phi_{2}\right)=$ $4 a_{23}\left(\phi_{2}-\phi_{3}\right)+2 b_{23}<0$, then $\phi_{\text {infl }}=\phi_{2}$, otherwise $\phi_{\text {infl }}=\phi_{3}$. 


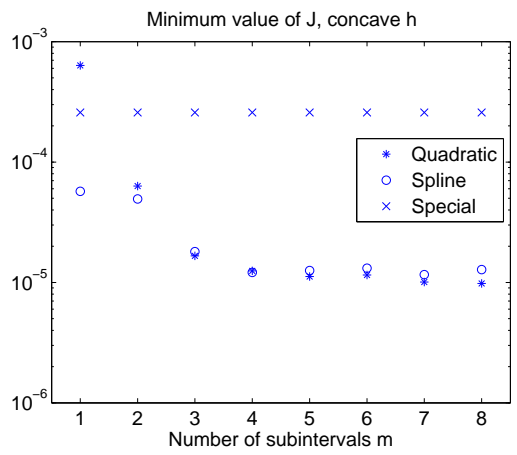

FIgURE 12. Values of $J$ in the minima of the quadratic program (40) for different number of subintervals for the estimation of $\hat{h}$.

- If $a \neq 0$ and $b \neq 0$, then compute the inflection point of $r_{23}$ :

$$
\phi_{\mathrm{inf}, 23}:=\frac{\phi_{2}+2 \phi_{3}}{3}+\frac{b_{23}}{3 a_{23}} ;
$$

note that $r_{23}^{\prime \prime \prime}\left(\phi_{\text {infl, } 23}\right)=6 a_{23}$.

- If $\left(\phi_{\text {infl,23 }} \leq \phi_{2}\right.$ and $\left.a_{23}<0\right)$ or $\left(\phi_{\text {infl, } 23} \geq \phi_{3}\right.$ and $\left.a_{23}>0\right)$, then $\phi_{\text {infl }}=\phi_{3}$.

- If $\left(\phi_{\text {infl,23 }} \geq \phi_{3}\right.$ and $\left.a_{23}<0\right)$ or $\left(\phi_{\text {infl, } 23} \leq \phi_{2}\right.$ and $\left.a_{23}>0\right)$, then $\phi_{\text {infl }}=\phi_{2}$.

- If $\phi_{\mathrm{inf}, 23} \in\left(\phi_{2}, \phi_{3}\right)$ and $a_{23}<0$, then $\bar{f}_{\mathrm{b}}$ has three inflection points; $\phi_{2}, \phi_{\mathrm{infl}, 23}$ and $\phi_{3}$. Then one may enlarge the interval $\left[\phi_{2}, \phi_{3}\right]$ (i.e., redefine $\phi_{2}$ and $\left.\phi_{3}\right)$ so that $r_{23}$ becomes a linear function.

- If $\phi_{\text {infl,23 }} \in\left(\phi_{2}, \phi_{3}\right)$ and $a_{23}>0$, then $\bar{f}_{\mathrm{b}}$ has a unique inflection point at $\phi_{\text {infl }}=\phi_{\text {infl, } 23}$.

6.3. The right interval. For the right interval $\left[\phi_{4}, \phi_{\max }\right]$, we note that $\phi_{\max }$ is not known a priori and the (convex) flux function should be nonnegative. An estimation of $\phi_{\max }$ can be obtained at the final steady state in either the Kynch or the Diehl test. However, for the type of flux functions with $f_{\mathrm{b}}^{\prime}\left(\phi_{\max }\right)=0$, which holds for the example flux (41), the steady state is not reach until $t \rightarrow \infty$. Furthermore, most of the published experimental batch tests are finished before the steady state arises.

If one had an estimation or guess of $\phi_{\max }$, of course a spline fit (e.g. similar to the one for the middle interval in Section 6.2) could be made; however we choose here the most simple alternative. A $C^{1}$ function $\bar{f}_{\mathrm{b}}$ is obtained by extending $\bar{f}_{\mathrm{b}}$ by a straight line through the point $\left(\phi_{4}, \breve{f}_{\mathrm{b}}\left(\phi_{4}\right)\right)$ with slope $\breve{f}_{\mathrm{b}}^{\prime}\left(\phi_{4}\right)$ :

$$
r_{45}(\phi):=\breve{f}_{\mathrm{b}}\left(\phi_{4}\right)+\breve{f}_{\mathrm{b}}^{\prime}\left(\phi_{4}\right)\left(\phi-\phi_{4}\right) .
$$

Note that (8) yields $\breve{f}_{\mathrm{b}}{ }^{\prime}\left(\phi_{4}\right)=\breve{f}_{\mathrm{b}}{ }^{\prime}\left(\phi_{h}\left(t_{\text {end }}\right)\right)=-h\left(t_{\text {end }}\right) / t_{\text {end }}<0$. Denote the intersection of this line with the $\phi$-axis by

$$
\phi_{5}:=\phi_{4}-\frac{\breve{f}_{\mathrm{b}}\left(\phi_{4}\right)}{\breve{f}_{\mathrm{b}}^{\prime}\left(\phi_{4}\right)} .
$$

This is an approximation of $\phi_{\max }$. 
6.4. Estimation from synthetic data. As a demonstration with the synthetic data from the two simulations above, one may decide, from inspection of Figures 6 and 12 , to choose the following methods for the different intervals:

$$
\begin{array}{ll}
{\left[0, \phi_{1}\right]:} & \text { quadratic polynomial }(56), \\
{\left[\phi_{1}, \phi_{2}\right]:} & \text { spline-fit method with one interval, } \\
{\left[\phi_{2}, \phi_{3}\right]:} & \text { cubic polynomial }(57) \text { or linear function, } \\
{\left[\phi_{3}, \phi_{4}\right]:} & \text { special-fit method with one interval, } \\
{\left[\phi_{4}, \phi_{5}\right]:} & \text { first-order polynomial }(58) .
\end{array}
$$

The Diehl and Kynch tests yield the numerical interval $\left[\phi_{2}, \phi_{3}\right]=[0.1361,0.2369]$, which for the middle interval means that $a_{23}=-24.15, b_{23}=0.6330$ and $\phi_{\text {infl,23 }}=$ 0.1946; see Section 6.2. Since $\phi_{2}<\phi_{\text {infl,23 }}<\phi_{3}$ and $a_{23}<0$, a cubic polynomial fit would yield three inflection points of the final $\bar{f}_{\mathrm{b}}$. Therefore, we enlarge the interval $\left[\phi_{2}, \phi_{3}\right]$ in both ends until we can fit a linear function so that $\bar{f}_{\mathrm{b}} \in C^{1}$ at the end points of this interval. After this procedure, we have the following values:

$$
\phi_{1}=0.0578, \quad \phi_{2}=0.1319, \quad \phi_{3}=0.2527, \quad \phi_{4}=0.5290 \quad \phi_{5}=0.6061 .
$$

These correspond to the five dashed vertical lines in Figure 13, which shows the graph of the following estimated flux function (for simplicity, numerical values are given by three significant figures):

$$
\bar{f}_{\mathrm{b}}(\phi)= \begin{cases}\phi(5.52-55.0(\phi-0.0578)), & 0 \leq \phi<0.0578 \\ -\phi\left(93.2 \tau_{12}(\phi)^{2}-52.1 \tau_{12}(\phi)+1.75\right) & 0.0578 \leq \phi<0.132 \\ 0.512-1.44 \phi, & 0.132 \leq \phi<0.253 \\ \phi\left(\frac{4.68 \cdot 10^{-5}}{\tau_{34}(\phi)^{3}}+\frac{0.0482}{\tau_{34}(\phi)^{2}}+0.00430\right), & 0.253 \leq \phi<0.529 \\ 0.0221-0.365 \phi, & 0.529 \leq \phi \leq 0.606\end{cases}
$$

where

$$
\begin{aligned}
\tau_{12}(\phi) & =0.838\left(2 \cos \left(\frac{\alpha_{12}(\phi)-2 \pi}{3}\right)+1\right) \\
\alpha_{12}(\phi) & =\arccos \left(1+2.96\left(0.989-\left(0.8+\frac{0.0500}{\phi}\right)\right)\right) \\
\tau_{34}(\phi) & =\frac{0.0482 \phi+\sqrt{0.00231 \phi^{2}+1.76 \cdot 10^{-5} \phi}}{0.250-0.269 \phi}
\end{aligned}
$$

Finally, we use the estimated flux function $\bar{f}_{\mathrm{b}}$ for numerical simulations of the Kynch and Diehl test, respectively; see Figure 14.

\section{Conclusions}

Given data from a Kynch batch settling test, there are two ways to determine an explicitly given flux function with respect to the representation formulas in Section 2.3. In Section 3, we start from functional forms of the curved discontinuity, denoted by $\breve{h}$, containing parameters to be determined and where the functional form is such that the inverse of $\breve{\eta}(t):=\breve{h}(t)-t \breve{h}^{\prime}(t)$ can be expressed as an explicit function so that eventually (14) can be an explicit expression for a smooth and 


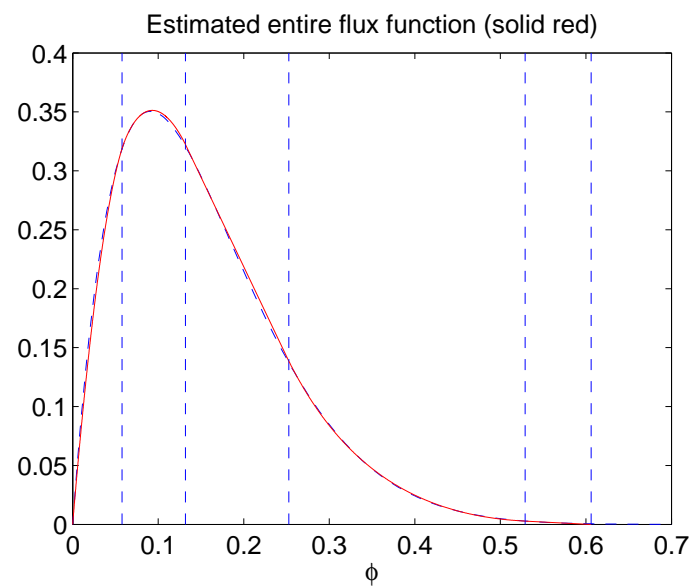

Figure 13. The entire estimated flux (59) (solid red line) and the known flux (41) (dashed blue).
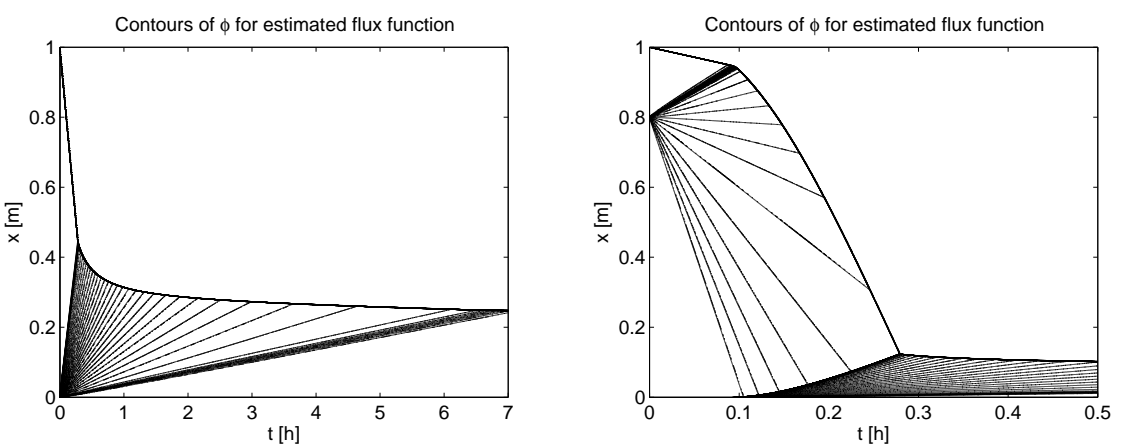

Figure 14. Contours of the numerical solution of a Kynch test (left) and Diehl test (right) when using the estimated flux function $\bar{f}_{\mathrm{b}}(59)$.

convex function $\breve{f}_{\mathrm{b}}$. An alternative treatment could consist in starting with functional forms of the settling velocity function $f_{\mathrm{b}}$, or equivalently $v_{\mathrm{s}}$, (rather than $\breve{h}$ ), and using (16) to get an expression with parameters, and which is fitted to data by a least-squares minimization. A version of the latter idea has been applied by Grassia et al. [36], who use a piecewise linear approach for the function $r(\phi)=(1-\phi)^{2} / v_{\mathrm{s}}(\phi)=\phi(1-\phi)^{2} / f_{\mathrm{b}}(\phi)$. The decisive difference in results consists in the observation that their approach leads to an estimated portion of the flux $\breve{f}_{\mathrm{b}}$ which is not convex; moreover, their identified portions of $f_{\mathrm{b}}$ are only piecewise differentiable and have slight but appreciable "kinks" at boundaries defined by the subintervals of piecewise definition of $r(\phi)$.

The main feature of the method presented herein consists in the fact that the approximations $\breve{h}$ and $\widehat{h}$ of the data points (19) arising from the Kynch and Diehl tests are provably convex and concave, respectively (cf. Theorem 4.1). This property ensures that the flux $f_{\mathrm{b}}$ identified can be directly used to determine exact solutions of (2). Moreover, one should keep in mind that the function $f_{\mathrm{b}}$ appears in 
models of continuous sedimentation, which are (roughly speaking) posed as conservation laws with the flux $f(\phi, t)=q(t) \phi-f_{\mathrm{b}}(\phi)$, where $q$ is a controllable positive or negative bulk velocity (see $[6,8,19,24]$ for details). Note carefully that $f(\phi, t)$ has the same second derivative (with respect to $\phi$ ) as $f_{\mathrm{b}}$. For the implementation of control strategies and numerical simulators, accurate knowledge of the location of the extrema of $f(\phi, t)$ (as a function of $\phi$ ) is very important. For instance, according to a theorem in [22] related to transient solutions, the maximum of the flux function is very important for the distinction whether overflow situations will occur or not (cf. the "safe" and "dangerous" regions in that paper). Reliable and efficient numerical methods for the numerical simulation of SSTs, for example those based on the Engquist-Osher scheme [28] (see [6, 8]), are likewise based on a reliable computation of these extrema.

Clearly, the present treatment has certain limitations. For example, while the Kynch and Diehl tests provide convex and concave portions of $f_{\mathrm{b}}$, respectively, one has to apply special care in gluing these portions together in such a way that the global flux has one inflection point only. Section 6 discusses this problem and presents an ad-hoc treatment of this problem. However, this problem is not inherent to the flux identification technique. Furthermore, the method presupposes that the suspension under study behaves indeed according the model (2), which means, for example, that effects of sediment compressibility are absent. These effects are, however, significant for suspensions that are flocculated, and which are described by (1) only for concentrations $\phi \leq \phi_{\mathrm{c}}$, where $\phi_{\mathrm{c}}$ is a material-dependent critical concentration or gel point at which particles touch each other. Nevertheless, in these cases the present treatment can still provide a valid starting point for flux identification. The authors plan to apply the present technique to a number of published and original experimental data.

Hyperbolic conservation laws of the form (1) have arisen in the macroscopic modelling of vehicle traffic flow for more than a century [46]. Of course, more sophisticated models have been developed; however, the need for model calibration is always present; see e.g. $[12,13,39,49,57]$ and references therein. For the identification of the "fundamental diagram", i.e. flux function $f_{\mathrm{b}}$ of $(1)$, we note that the upper part of the solution of the Diehl test (see Figure 7, right) can be interpreted as the event when vehicles in a queue of length $H-H_{0}$ start to drive as the red light at $x=H_{0}$ turns into green at $t=0$. Thus, the techniques presented here may also be employed in applications other than sedimentation.

\section{Appendix A. An inverse of the CUbic polynomial (32)}

The three roots of the cubic equation (32) can be found in the following way according to Cardano's method. Skipping the index $i$, the equation is

$$
-2 a t^{3}-b t^{2}+d=y \quad \text { where } a<0, b>0 \text { and } d>y .
$$

Setting $s:=t+\frac{b}{6 a}$ one gets the equation

$$
s^{3}+p s+q=0, \quad \text { where } \quad p:=-\frac{b^{2}}{12 a^{2}}<0 \quad \text { and } \quad q:=\frac{b^{3}}{108 a^{3}}+\frac{y-d}{2 a} .
$$

Introducing two new variables $u$ and $v$ via $u+v=s$ and $3 u v+p=0$, one gets

$$
u^{3}+v^{3}+q=0, \quad u^{6}+q u^{3}-\frac{p^{3}}{27}=0, \quad v^{6}+q v^{3}-\frac{p^{3}}{27}=0
$$


The second and third equations in (61) are second-order equations in $u^{3}$ and $v^{3}$, respectively, both having the discriminant

$$
D:=\frac{q^{2}}{4}+\frac{p^{3}}{27}
$$

We shall prove that $D<0$. The possible interval for $y$ in (32) yields (for all three roots)

$$
d-\frac{b^{3}}{27 a^{2}}<y<d \quad \Longleftrightarrow \quad \frac{b^{3}}{108 a^{3}}<\frac{y-d}{2 a}+\frac{b^{3}}{108 a^{3}}<-\frac{b^{3}}{108 a^{3}} \Longleftrightarrow|q|<-\frac{b^{3}}{108 a^{3}} .
$$

Hence

$$
D<\frac{1}{4}\left(\frac{b^{3}}{108 a^{3}}\right)^{2}-\frac{1}{27}\left(\frac{b^{2}}{12 a^{2}}\right)^{3}=0 .
$$

This means that the equations (61) are satisfied by

$$
u^{3}=-\frac{q}{2}+\mathrm{i} R, \quad v^{3}=-\frac{q}{2}-\mathrm{i} R,
$$

where $\mathrm{i}=\sqrt{-1}$ and $R:=\sqrt{-D}>0$. Let $\alpha$ denote the argument of the complex number in the right-hand side of the first equation of (62). Note that $\left|u^{3}\right|=(-p / 3)^{3 / 2}$. Hence, we have $0<\alpha<\pi$ and

$$
\alpha=\arccos \left(-\frac{q}{2(-p / 3)^{3 / 2}}\right)=\arccos \left(1-\frac{54 a^{2}(d-y)}{b^{3}}\right) .
$$

Then the solutions (62) are

$$
u_{k}=\mathrm{e}^{\mathrm{i}(\alpha+k 2 \pi) / 3} \sqrt{-\frac{p}{3}}, \quad k=-1,0,1 ; \quad v_{k}=\mathrm{e}^{\mathrm{i}(-\alpha+k 2 \pi) / 3} \sqrt{-\frac{p}{3}}, \quad k=-1,0,1 .
$$

These should be paired so that $3 u v+p=0$, i.e.,

$$
u_{0} v_{0}=-\frac{p}{3}, \quad u_{-1} v_{1}=-\frac{p}{3}, \quad u_{1} v_{-1}=-\frac{p}{3} .
$$

Substituting back and noting that

$$
\sqrt{-\frac{p}{3}}=-\frac{b}{6 a}
$$

the three solutions of (60) are

$$
\begin{aligned}
& t_{1}=s-\frac{b}{6 a}=u_{0}+v_{0}-\frac{b}{6 a}=-\frac{b}{6 a}\left(2 \cos \left(\frac{\alpha}{3}\right)-1\right) \\
& t_{2}=s-\frac{b}{6 a}=u_{-1}+v_{1}-\frac{b}{6 a}=-\frac{b}{6 a}\left(2 \cos \left(\frac{\alpha}{3}-\frac{2 \pi}{3}\right)+1\right) \\
& t_{3}=s-\frac{b}{6 a}=u_{1}+v_{-1}-\frac{b}{6 a}=-\frac{b}{6 a}\left(2 \cos \left(\frac{\alpha}{3}+\frac{2 \pi}{3}\right)+1\right) .
\end{aligned}
$$

If $q<0$, (63) gives that $\cos \alpha>0$ and $0<\alpha<\pi / 2$. Then one can conclude that $t_{2}$ is the middle root. Analogously, the case $q \geq 0$ yields that $t_{2}$ is the middle root. Substituting back we get the solution in the original parameters, and hence the inverse (33). 
BÜRGER AND DIEHL

\section{ACKNOWLEDGEMENTS}

R. Bürger acknowledges partial support by Fondecyt project 1090456, BASAL project CMM, Universidad de Chile and Centro de Investigación en Ingeniería Matemática ( $\left.\mathrm{CI}^{2} \mathrm{MA}\right)$, Universidad de Concepción, and CONICYT project Anillo ACT1118 (ANANUM).

\section{REFERENCES}

[1] D.P. Ballou. Solutions to nonlinear hyperbolic Cauchy problems without convexity conditions. Trans. Amer. Math. Soc., 152:441-460, 1970.

[2] J.L. Bueno, J. Coca, E. Cuesta, A. Gutierrez Lavin, and G. Velasco. Sedimentation of coal slurries: A procedure for the determination of the flocculated-solid flux curve useful for the design of continuous settling tanks. Powder Tech., 63:133-140, 1990.

[3] R. Bürger, A. Coronel, and M. Sepúlveda. A numerical descent method for an inverse problem of a scalar conservation law modelling sedimentation. In: K. Kunisch, G. Of, and O. Steinbach, editors, Numerical Mathematics and Advanced Applications. Proceedings of ENUMATH 2007, the 7th European Conference on Numerical Mathematics and Advanced Applications, Graz, Austria, September 2007, pages 225-232. Springer-Verlag, Berlin, 2008.

[4] R. Bürger, A. Coronel, and M. Sepúlveda. Numerical solution of an inverse problem for a scalar conservation law modelling sedimentation. In: E. Tadmor, J.-G. Liu, and A.E. Tzavaras, editors, Hyperbolic Problems: Theory, Numerics and Applications. Proceedings of Symposia in Applied Mathematics, Vol. 67, Part 2, pages 445-454. American Mathematical Society, Providence, RI, USA, 2009.

[5] R. Bürger, S. Diehl, and I. Nopens. A consistent modelling methodology for secondary settling tanks in wastewater treatment. Water Res., 45:2247-2260, 2011.

[6] R. Bürger, K.H. Karlsen, N.H. Risebro, and J.D. Towers. Well-posedness in $B V_{t}$ and convergence of a difference scheme for continuous sedimentation in ideal clarifier-thickener units. Numer. Math., 97:25-65, 2004.

[7] R. Bürger, K.H. Karlsen, H. Torres, and J.D. Towers. Second-order schemes for conservation laws with discontinuous flux modelling clarifier-thickener units. Numer. Math., 116:579-617, 2010.

[8] R. Bürger, K.H. Karlsen, and J.D. Towers. A mathematical model of continuous sedimentation of flocculated suspensions in clarifier-thickener units. SIAM J. Appl. Math., 65:882-940, 2005.

[9] R. Bürger and E.M. Tory. On upper rarefaction waves in batch settling. Powder Technol., 108:74-87, 2000

[10] R. Bürger and W.L. Wendland. Sedimentation and suspension flows: Historical perspective and some recent developments. J. Eng. Math., 41:101-116, 2001.

[11] M.C. Bustos, F. Concha, R. Bürger, and E.M. Tory. Sedimentation and Thickening, Kluwer Academic Publishers, Dordrecht, 1999.

[12] N. Chiabaut, C. Buisson, and L. Leclercq. Fundamental diagram estimation through passing rate measurements in congestion. Intelligent Transport. Systems, IEEE Transactions on, 10(2):355-359, june 2009.

[13] N. Chiabaut and L. Leclercq. Wave velocity estimation through automatic analysis of cumulative vehicle count curves. Transport. Res. Record, (2249):1-6, 2011.

[14] A. Coronel, F. James, and M. Sepúlveda. Numerical identification of parameters for a model of sedimentation processes. Inverse Problems, 19(4):951-972, 2003.

[15] J.W. Daniel. Stability of the solution of definite quadratic programs. Mathematical Programming, 5:41-53, 1973

[16] P.J.T. Dankers and J.C. Winterwerp. Hindered settling of mud flocs: theory and validation. Cont. Shelf Res., 27:1893-1907, 2007.

[17] G.B. Dantzig, J. Folkman, and N. Shapiro. On the continuity of the minimum set of a continuous function. J. Math. Anal. Appl., 17(3):519-548, 1967.

[18] J. De Clercq, M. Devisscher, I. Boonen, J. Defrancq, and P.A. Vanrolleghem. Analysis and simulation of the sludge profile dynamics in a full-scale clarifier. J. Chem. Technol. Biotechnol., 80:523-530, 2005. 
[19] S. Diehl. A conservation law with point source and discontinuous flux function modelling continuous sedimentation. SIAM J. Appl. Math., 56(2):388-419, 1996.

[20] S. Diehl. Dynamic and steady-state behaviour of continuous sedimentation. SIAM J. Appl. Math., 57(4):991-1018, 1997.

[21] S. Diehl. Estimation of the batch-settling flux function for an ideal suspension from only two experiments. Chem. Eng. Sci., 62:4589-4601, 2007.

[22] S. Diehl. Operating charts for continuous sedimentation IV: limitations for control of dynamic behaviour, J. Eng. Math., 60:249-264, 2008.

[23] S. Diehl. A regulator for continuous sedimentation in ideal clarifier-thickener units. J. Eng. Math., 60:265-291, 2008.

[24] S. Diehl. The solids-flux theory-confirmation and extension by using partial differential equations. Water Res., 42:4976-4988, 2008.

[25] S. Diehl. Shock-wave behaviour of sedimentation in wastewater treatment: A rich problem. In K. Åström, L.-E. Persson, and S.D. Silvestrov, editors, Analysis for Science, Engineering and Beyond, volume 6 of Springer Proceedings in Mathematics, pages 175-214. Springer Berlin Heidelberg, 2012.

[26] P. Diplas and A.N. Papanicolaou. Batch analysis of slurries in zone settling regime. J. Eviron. Engrg., 123:659-667, 1997.

[27] A. Einstein. Eine neue Bestimmung der Moleküldimensionen. Ann. d. Phys., 19:289-306, 1906, and A. Einstein. Berichtigung zu meiner Arbeit: "Eine neue Bestimmung ...". Ann. d. Phys., 34:591-592, 1911.

[28] B. Engquist and S. Osher. One-sided difference approximations for nonlinear conservation laws. Math. Comp., 36:321-351, 1981.

[29] C. Eswaraiah, S.K. Biswal, and B.K. Mishra. Settling characteristics of ultrafine ore slimes. Int. J. Minerals, Metallurgy and Materials, 19:95-99, 2012.

[30] E.M. Fernández-Berdaguer and G.B. Savioli. An inverse problem arising from the displacement of oil by water in porous media. Applied Num. Math., 59(10):2452-2466, 2009.

[31] B. Fitch. Thickening theories - an analysis. AIChE J., 39(1):27-36, 1993.

[32] R. Font. Analysis of the batch sedimentation test, Chem. Eng. Sci., 46:2473-2482, 1991.

[33] R. Font, P. García, and M. Pérez. Analysis of the variation of the upper discontinuity in sedimentation batch test. Sep. Sci. Technol., 33:1487-1510, 1998.

[34] R. Font and M.L. Laveda. Semi-batch test of sedimentation. Application to design. Chem. Eng. J., 80:157-165, 2000.

[35] P. Grassia, S.P. Usher, and P.J. Scales. A simplified parameter extraction technique using batch settling data to estimate suspension material properties in dewatering applications. Chem. Eng. Sci., 63:1971-1986, 2008.

[36] P. Grassia, S.P. Usher, and P.J. Scales. Closed-form solutions for batch settling height from model settling flux functions. Chem. Eng. Sci., 66:964-972, 2011.

[37] P.G.W. Hawksley. The effect of concentration on the settling of suspensions and flow through porous media. In: Some Aspects of Fluid Flow, E. Arnold \& Co., London 1951, pp. 114-135.

[38] E. Iritani, T. Hashimoto, and N. Katagiri. Gravity consolidation-sedimentation behaviors of concentrated $\mathrm{TiO}_{2}$ suspensions. Chem. Eng. Sci., 64:4414-4423, 2009.

[39] P. Jaisson and F. De Vuyst. Data assimilation and inverse problem for fluid traffic flow models and algorithms. Int. J. Num. Meth. Eng., 76(6):837-861, 2008.

[40] F. James and M. Sepúlveda. Convergence results for the flux identification in a scalar conservation law. SIAM J. Control Optim., 37(3):869-891, 1999.

[41] H. Kang and K. Tanuma. Inverse problems for scalar conservation laws. Inverse Problems, 21(3):1047-1059, 2005.

[42] R.D. Karamisheva and M.A. Islam. Development of a new model for batch sedimentation and application to secondary settling tanks design. Water Environ. Res., 77:3066-3073, 2005.

[43] M. Kunik. A solution formula for a non-convex scalar hyperbolic conservation law with monotone initial data. Math. Meth. Appl. Sci., 16(12):895-902, 1993.

[44] G.J. Kynch. A theory of sedimentation. Trans. Faraday Soc., 48:166-176, 1952.

[45] D.R. Lester, S.P. Usher, and P.J. Scales. Estimation of the hindered settling function $R(\phi)$ from batch-settling tests. AIChE J., 51:1158-1168, 2005.

[46] M.J. Lighthill and G.B. Whitham. On kinematic waves. II. A theory of traffic flow on long crowded roads. Proc. Royal Soc. London, 229(1178):317-345, 1955. 
[47] Z. Li and D. Zhang. A model for characterizing the process of activated sludge compression settling. Adv. Mat. Res., 518-523:3680-3686, 2012.

[48] J. Mutrynowska and B. Grzegorzewski. Optical analysis of red blood cell sediment formation. Biorheology, 44:285-297, 2007.

[49] D. Ngoduy and M.J. Maher. Calibration of second order traffic models using continuous cross entropy method. Transport. Res. Part C: Emerging Technologies, 24(0):102-121, 2012.

[50] O.A. Oleinik. Uniqueness and stability of the generalized solution of the Cauchy problem for a quasi-linear equation. Uspekhi Mat. Nauk, 14:165-170, 1959. Amer. Math. Soc. Trans. Ser. 2, 33, (1964), pp. 285-290.

[51] A.N. Papanicolaou and A.R. Maxwell. Methodological considerations for studying self-weight fluidization in a sedimentation column. Int. J. Mineral Process., 78:140-152, 2006.

[52] J.F. Richardson and W.N. Zaki. The sedimentation of a suspension of uniform spheres under conditions of viscous flow. Chem. Eng. Sci., 3:65-73, 1954.

[53] G.B. Savioli and E.M. Fernández-Berdaguer. The estimation of oil water displacement functions. Latin American Applied Res., 37(3):187-194, 2007.

[54] H.H. Steinour. Rate of sedimentation. Concentrated flocculated suspensions of powders. Ind. Eng. Chem., 36(10):901-907, 1944.

[55] H.H. Steinour. Rate of sedimentation. Nonflocculated suspensions of uniform spheres. Ind. Eng. Chem., 36(7):618-624, 1944.

[56] A.-E. Stricker, I. Takács, and A. Marquot. Hindered and compression settling: parameter measurement and modelling. Wat. Sci. Tech., 56(12):101-110, 2007.

[57] M. Treiber, A. Kesting, and D. Helbing. Three-phase traffic theory and two-phase models with a fundamental diagram in the light of empirical stylized facts. Transport. Res. Part B: Methodological, 44(8-9):983-1000, 2010.

[58] V. Vand. Viscosity of solutions and suspensions. I. Theory. J. Phys. Chem., 52:277-299, 1948.

[59] A. Vanderhasselt and P.A. Vanrolleghem. Estimation of sludge sedimentation parameters from single batch settling curves. Wat. Res., 34(2):395-406, 2000.

[60] P.A. Vesilind. Treatment and Disposal of Wastewater Sludges. Ann Arbor Science Publishers, Ann Arbor, Michigan, USA, 1974.

[61] A.G. Waters and K.P. Galvin. Theory and application of thickener design. Filtrat. Separ., 28:110-116, 1991. 